Research Article

\title{
Based on Integrated Bioinformatics Analysis Identification of Biomarkers in Hepatocellular Carcinoma Patients from Different Regions
}

\author{
Linxin Teng, Kaiyuan Wang, Yu Liu, Yanxia Ma, Weiping Chen, and Lei Bi \\ School of Preclinical Medicine, Nanjing University of Chinese Medicine, 138 Xianlin Road, Nanjing, Jiangsu 210023, China \\ Correspondence should be addressed to Lei Bi; bella_nj@163.com
}

Received 24 July 2019; Accepted 24 September 2019; Published 28 October 2019

Guest Editor: Bin Duan

Copyright (c) 2019 Linxin Teng et al. This is an open access article distributed under the Creative Commons Attribution License, which permits unrestricted use, distribution, and reproduction in any medium, provided the original work is properly cited.

\begin{abstract}
Accumulating statistics have shown that liver cancer causes the second highest mortality rate of cancer-related deaths worldwide, of which $80 \%$ is hepatocellular carcinoma (HCC). Given the underlying molecular mechanism of HCC pathology is not fully understood yet, identification of reliable predictive biomarkers is more applicable to improve patients' outcomes. The results of principal component analysis (PCA) showed that the grouped data from 1557 samples in Gene Expression Omnibus (GEO) came from different populations, and the mean tumor purity of tumor tissues was 0.765 through the estimate package in R software. After integrating the differentially expressed genes (DEGs), we finally got 266 genes. Then, the protein-protein interaction (PPI) network was established based on these DEGs, which contained 240 nodes and 1747 edges. FOXM1 was the core gene in module 1 and highly associated with FOXM1 transcription factor network pathway, while FTCD was the core gene in module 2 and was enriched in the metabolism of amino acids and derivatives. The expression levels of hub genes were in line with The Cancer Genome Atlas (TCGA) database. Meanwhile, there were certain correlations among the top ten genes in the up- and downregulated DEGs. Finally, Kaplan-Meier curves and receiver operating characteristic (ROC) curves were plotted for the top five genes in PPI. Apart from $\mathrm{CDKN} 3$, the others were closely concerned with overall survival. In this study, we detected the potential biomarkers and their involved biological processes, which would provide a new train of thought for clinical diagnosis and treatment.
\end{abstract}

\section{Introduction}

Liver cancer is highly fatal, which causes the second highest death rate of cancer-related mortality worldwide [1,2]. Globally, it is estimated that approximately $80 \%$ of liver cancers were HCC [3]. Nobody disputes that this is a public health challenge that needs widespread attention. HCC is a multigene disease caused by the interaction of multiple cancer-promoting and suppressing genes with the microenvironment, and its molecular mechanism is still unclear. Thus, the identification of new potential therapeutic targets is urgently needed.

In recent years, despite the advances in our knowledge of the genetic factors, it is a pity that the death rates were increasing rapidly $[4,5]$. If the HCC patients can be diagnosed early, the survival rate may be greatly improved by means of liver resection $[6,7]$. However, due to the late diagnosis of most patients with HCC, the physical condition is not good enough to withstand the risk of surgery $[8,9]$. What is worse, the survival rate of patients with advanced HCC is further decreased due to the widespread resistance to chemotherapy. Sorafenib, for example, a multikinase inhibitor, is widely used for the treatment of patients with advanced HCC with a long application time [10,11], but patients invariably develop sorafenib resistance and it only provides limited survival benefit [12]. As a result, we are badly in need of finding new diagnostic and prognostic markers for HCC, which might facilitate early diagnosis and guide treatment decisions to improve patients' survival and quality of life.

In this study, we downloaded the expression matrix of six datasets from the Gene Expression Omnibus (GEO) database, including 630 adjacent normal and 927 tumor tissues. PCA, tumor purity evaluation, and differential expression gene 
(DEG) analysis were performed by using R software. 266 DEGs were finally obtained, consisting of 81 upregulated and 185 downregulated genes. FunRich undertook the entire enrichment analyses in our experiment, while Cytoscape was employed to build a network diagram. We had found that upregulated genes were closely related to mitotic cell cycle. Different from upregulated genes, downregulated genes were enriched in lipid and lipoprotein derivative pathway. To further explore the role of these DEGs, we divided the PPI network into several independent modules. FOXM1 and FTCD were core genes in two separate models with the highest score, respectively. The former is enriched in FOXM1 transcription factor network pathway, while the latter is mainly enriched in the metabolism of amino acid and derivative pathway. Finally, The Cancer Genome Atlas (TCGA) data were used to test our results and predict overall survival related to five hub genes in PPI. We found certain correlations among hub genes, which might reveal potential signaling pathways in HCC. And 4 of 5 hub genes were connected with low overall survival of HCC patients. Undoubtedly, recognition of biomarkers in HCC that plays a key role in disease progression can provide new insights into the development, prognosis, and treatment of HCC.

\section{Materials and Methods}

2.1. Tumor Purity Estimation and Differential Expression Gene Analysis. All the gene expression profile data originated from the GEO database (https://www.ncbi.nlm.nih.gov/geo). There were huge liver cancer mRNA microarray datasets in the GEO database, and the included datasets need to meet the following conditions: (1) the microarray data were available; (2) they contained at least 100 samples; and (3) they employed tumor and adjacent normal tissues. Therefore, we selected the following datasets: GSE25097, GSE36376, GSE45436, GSE54236, GSE64041, and GSE112790. GEOquery package in R/Bioconductor software (version 3.6.1, https://www.r-project.org) was used to get datasets, which was applied to download gene expression and probe annotation information for the selected datasets. Then, the estimate package was used to estimate tumor purity, while the limma package for data normalization and gene differential expression matrix acquisition. In the differential expression gene analysis, $\mathrm{FDR}<0.05$ and $\log _{2} \mathrm{FC} \mid \geq 1$ were considered to be significant DEGs, which were visualized by ggplot2 package.

2.2. Enrichment Analysis. We divided the DEGs into two categories and ranked them in descending order of absolute values. Since the six datasets were not from the same platform, we used RRA package to integrate the DEGs. FunRich (version 3.1.3, http://www.funrich.org) is such powerful stand-alone software that we primarily used to perform functional enrichment analysis [13]. Biological process, biological pathway, cellular component, and molecular function can be achieved by FunRich in the present study.

2.3. PPI Network and Module Analysis. The Search Tool for the Retrieval of Interacting Genes database (STRING, https:// string-db.org) can provide information on protein interactions, whose data mainly came from structural predictions and literature reports [14]. Combined score $\geq 0.4$ was considered as the cutoff value, and the filtered node information was saved locally for subsequent visualization. Then, we used Cytoscape software (version 3.6.0, https://cytoscape.org) to build the protein-protein interaction (PPI) network and one of the plugin in Cytoscape named Molecular Complex Detection (MCODE) was applied to detect notable modules in this PPI network [15]. As is known to all, network modules, as one of the characteristics of protein networks, may have specific biological significance. The default advanced option parameters (degree cutoff $=2$, node score cutoff $=0.2$, and $\mathrm{k}$-core $=2$ ) in MCODE already met our requirements, so we did not modify it. Moreover, models with score $\geq 5$ were used for further path enrichment analysis, which can help to explore the potential biological functions of DEGs.

\subsection{Analysis for Expression Level and Correlation of the Hub} Genes. The Gene Expression Profiling Interactive Analysis (GEPIA, http://gepia.cancer-pku.cn) is an online website tool that can perform analysis including gene expression analysis and correlation analysis [16]. Data from TCGA and the Genotype-Tissue Expression (GTEx, http:// commonfund.nih.gov/GTEx/) were used to apply a standard processing pipeline before being used by GEPIA. Based on the huge amount of data from GEPIA, we used it to demonstrate the expression of hub genes in LIHC tissues and normal ones and then made a boxplot to visualize the results. There are three correlation coefficients (Pearson, Spearman, and Kendall) for users to choose in GEPIA, and any sets given by TCGA and/or GTEx expression data were used to check the relative ratios between two genes.

\subsection{Overall Survival Analysis and ROC Curve Analysis of Hub} Genes. Kaplan-Meier plotter (KM plotter, http://kmplot. com/analysis/) is a database that can be accessed openly, which is the largest dataset including breast, ovarian, lung, and gastric cancer [17]. This database is rich in gene expression data and overall survival information from TCGA, which we can use to draw survival curves with $95 \%$ confidence interval hazard ratio and log-rank $P$ value. Receiver operating characteristic (ROC) curve analysis was employed to verify the diagnostic performance of hub genes, and 3 years was set as the predicted time. Multivariate Cox proportional hazards regression analysis was performed based on hub genes. The risk score for predicting overall survival was calculated as follows: risk score $=\sum_{i=1}^{n}\left(\operatorname{coef}_{i} * \operatorname{Expr}_{i}\right)$, where coef is the regression coefficient and Expr is the expression level of the gene. Then, according to the mean risk score, samples were divided into low- and high-risk groups. Finally, survival analysis and ROC curve analysis of the risk score were performed by using the same method as described above.

\section{Results}

3.1. Tumor Purity Estimation of Tumor Tissue in Datasets and Identification of DEGs. 630 normal and 927 tumor samples were selected in this study (Table 1). The results of PCA showed 
TABLE 1: The gene expression profile data characteristics.

\begin{tabular}{|c|c|c|c|c|c|c|}
\hline Record & Organism & Tissue & $\begin{array}{c}\text { Normal } \\
(n=630)\end{array}$ & $\begin{array}{l}\text { Tumor } \\
(n=927)\end{array}$ & Region & Platform \\
\hline GSE25097 & $\begin{array}{l}\text { Homo } \\
\text { sapiens }\end{array}$ & $\begin{array}{l}\text { Liver } \\
\text { tumor }\end{array}$ & $243(38.6 \%)$ & $268(28.9 \%)$ & Boston & $\begin{array}{l}\text { GPL10687-Rosetta/Merck Human RSTA } \\
\text { Affymetrix } 1.0 \text { microarray, custom CDF }\end{array}$ \\
\hline GSE36376 & $\begin{array}{l}\text { Homo } \\
\text { sapiens }\end{array}$ & $\begin{array}{l}\text { Liver } \\
\text { tumor }\end{array}$ & $193(30.6 \%)$ & $240(25.9 \%)$ & Seoul & $\begin{array}{c}\text { GPL10558-Illumina HumanHT-12 V4.0 } \\
\text { expression beadchip }\end{array}$ \\
\hline GSE45436 & $\begin{array}{l}\text { Homo } \\
\text { sapiens }\end{array}$ & $\begin{array}{l}\text { Liver } \\
\text { tumor }\end{array}$ & $39(6.2 \%)$ & $95(10.2 \%)$ & Taipei & $\begin{array}{c}\text { GPL570_[HG-U133_Plus_2] Affymetrix Human } \\
\text { Genome U133 Plus 2.0 Array }\end{array}$ \\
\hline GSE54236 & $\begin{array}{l}\text { Homo } \\
\text { sapiens }\end{array}$ & $\begin{array}{l}\text { Liver } \\
\text { tumor }\end{array}$ & $80(12.7 \%)$ & $81(8.7 \%)$ & Modena & $\begin{array}{c}\text { GPL6480-Agilent-014850 Whole Human } \\
\text { Genome Microarray 4x44K G4112F (Probe Name } \\
\text { version) }\end{array}$ \\
\hline GSE64041 & $\begin{array}{l}\text { Homo } \\
\text { sapiens }\end{array}$ & $\begin{array}{l}\text { Liver } \\
\text { tumor }\end{array}$ & $60(9.5 \%)$ & $60(6.5 \%)$ & Basel & $\begin{array}{l}\text { GPL6244-[HuGene-1_0-st] Affymetrix Human } \\
\text { Gene 1.0 ST Array [transcript (gene) version] }\end{array}$ \\
\hline GSE112790 & $\begin{array}{l}\text { Homo } \\
\text { sapiens }\end{array}$ & $\begin{array}{l}\text { Liver } \\
\text { tumor }\end{array}$ & $15(2.4 \%)$ & $183(19.7 \%)$ & Tokyo & $\begin{array}{l}\text { GPL570_- [HG-U133_Plus_2] Affymetrix Human } \\
\text { Genome U133 Plus 2.0 Array }\end{array}$ \\
\hline
\end{tabular}

that the normal control group and the tumor group in the six datasets could be discriminated very well (Figures 1(a)-1(f)). Then, we calculated the tumor purity of 927 liver tumor tissues through the estimate algorithm. As shown in Figures $1(\mathrm{~g})$ and 1 (h), the purity of tumors ranged from 0.179 to 0.979 and $55.8 \%$ of the tumor samples had a greater value than the mean value of 0.765. After performing differential expression gene analysis on each dataset, 81 upregulated and 185 downregulated genes were finally detected by RRA (Supplementary Table S1). Compared with the adjacent normal ones, the expression of these genes in tumor tissues was all upregulated or downregulated $\geq 2$-folds (Figures 2(a)-2(f)). We sorted the upregulated and downregulated genes in ascending order according to the FDR values and created the heatmap with the top 20 genes (Figure 2(g)).

3.2. Enrichment Analysis of DEGs. DEGs from six independent datasets were integrated and introduced into FunRich for enrichment analysis. The biological processes for upregulated genes were mainly associated with spindle assembly and cell cycle (Figure 3(a) and Supplementary Table S2), while the molecular functions were about protein binding and kinase binding (Figure 3(b) and Supplementary Table S2). In addition, the vital gene of upregulated genes was called FOXM1, which was highly correlated with transcription factor activity and FOXM1 transcription factor network (Supplementary Table S2). The functional enrichment of downregulated genes was associated with metabolism, catalytic activity, and energy pathways (Figures 3(c) and 3(d)). And the pivotal gene FTCD in this group was enriched in methyltransferase activity, energy pathways, and histidine catabolism. Through the biological pathway enrichment analysis, we found that upregulated genes were closely related to mitotic cell cycle, DNA replication, mitotic G1-G1/S phases, and ATM pathway (Figure 4(a) and Supplementary Table S2). Different from upregulated genes, downregulated genes were enriched in lipid and lipoprotein derivative pathway (Figure 4(b) and Supplementary Table S2).

3.3. PPI Network Establishment and Pathway Analysis of Network Module. After introducing the gene list into STRING website, we finally got the information of 240 nodes and 1747 edges (combined score $\geq 0.4$ ). Then, the network diagram was presented by Cytoscape based on the STRING database (Figure 5(a) and Supplementary Table S3). Interestingly, most of the nodes with higher connectivity were upregulated genes, which signified that they would be closer to the center of the circle. Four modules with score $\geq 5$ were detected via MCODE (Figures 5(b)-5(e)). It can be seen in Figure 5(b) that the hub nodes were FOXM1, CCNA2, AURKA, CDKN3, and CDC20 in module 2. Besides, as shown in Figure 5(c), FTCD, HRG, AGXT, C8A, and TAT were nodes with highest connectivity in module 2 . Among the four models, only model 3 contained both of the protein nodes expressed by up- and downregulated genes, including AFP, PLG, CRP, FABP1, and SPP1. And the results of the pathway analysis for the two modules with the highest combined score are shown in Figures 5(f) and 5(g) and Supplementary Table S4. It is worth mentioning the most significant pathways in module 1 and module 2 were mitotic cell cycle and phase 1-functionalization of compounds, respectively.

3.4. Expression Level and Correlation of Hub Genes. A total of 419 samples were selected for gene expression level analysis, including 369 tumor tissues and 50 normal liver tissues. As shown in Figures 6(a)-6(e), the expression of the five hub genes in cancer tissues was significantly higher than that in normal ones. Moreover, it turned out by the correlation analysis that the increased expression of these genes was strongly correlated with the decreased expression of FTCD (Figures 6(f)-6(j)), and heatmap of correlation coefficients between hub genes was shown in Figure 6(k).

3.5. Prognostic Factor Detection and Validation. The analysis of 364 HCC patients clearly showed that 4 out of 5 genes with the highest degree in PPI had a significant impact on prognosis (Figure 7). High expression of FOXM1 ( $\mathrm{HR}=1.68$; $P=0.0033), \quad$ CCNA2 $(\mathrm{HR}=1.69 ; \quad P=0.0029), \quad$ AUPKA $(\mathrm{HR}=1.61 ; P=0.0069), \mathrm{CDKN} 3(\mathrm{HR}=1.29 ; P=0.15)$, and CDC20 $(\mathrm{HR}=2.3 ; P=3.4 e-06)$ indicated worse survival rate in patients with HCC. In ROC curve analysis, FOXM1 $(\mathrm{AUC}=0.654$, sensitivity $=0.533$, and specificity $=0.688)$, 


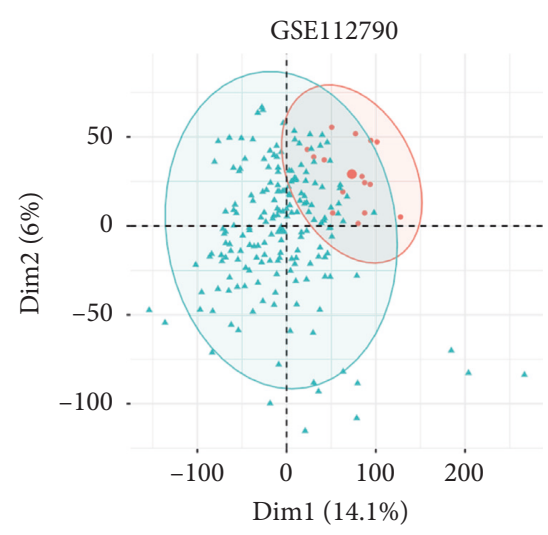

- Normal

$\triangle$ Tumor

(a)

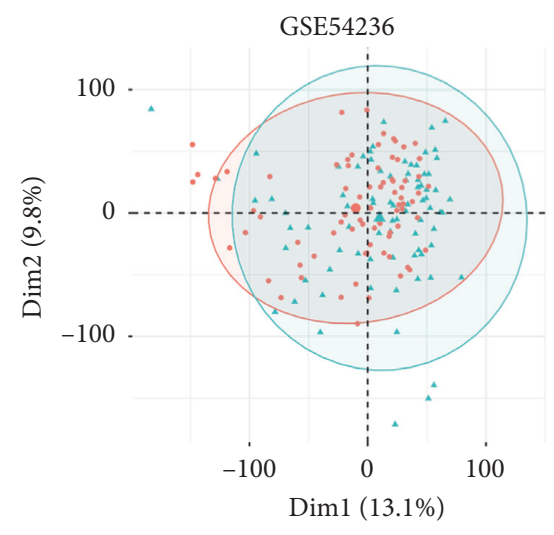

- Normal

$\triangle$ Tumor

(c)

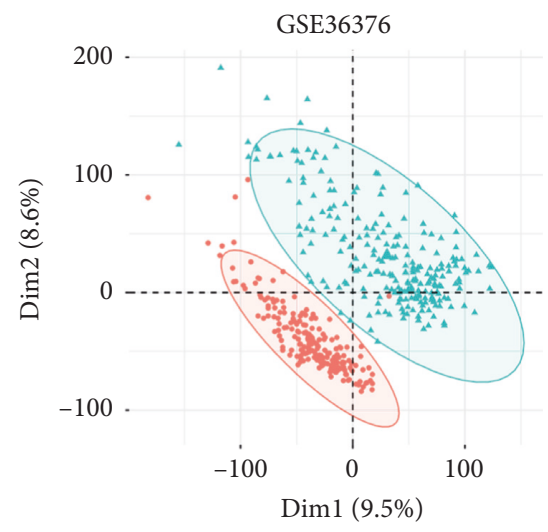

- Normal

$\triangle$ Tumor

(e)

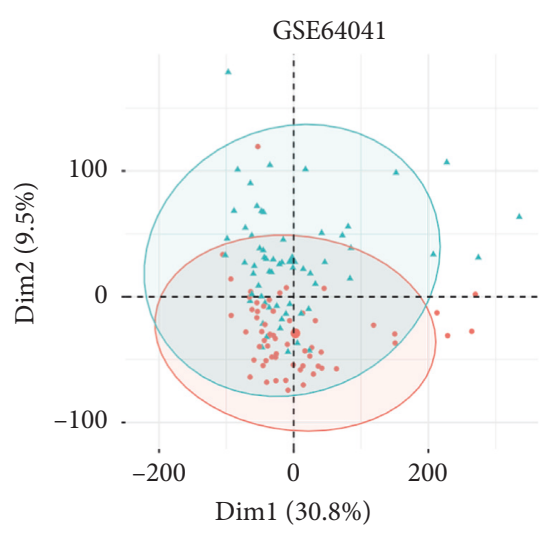

$\square$ Normal
$\square$ Tumor

(b)

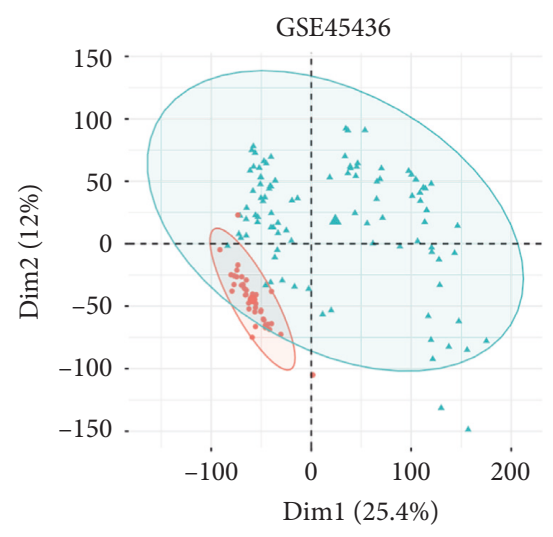

- Normal

$\triangle$ Tumor

(d)

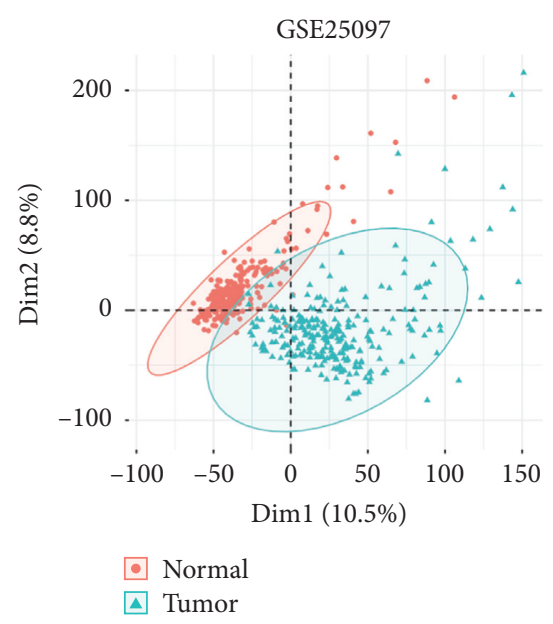

(f)

Figure 1: Continued. 

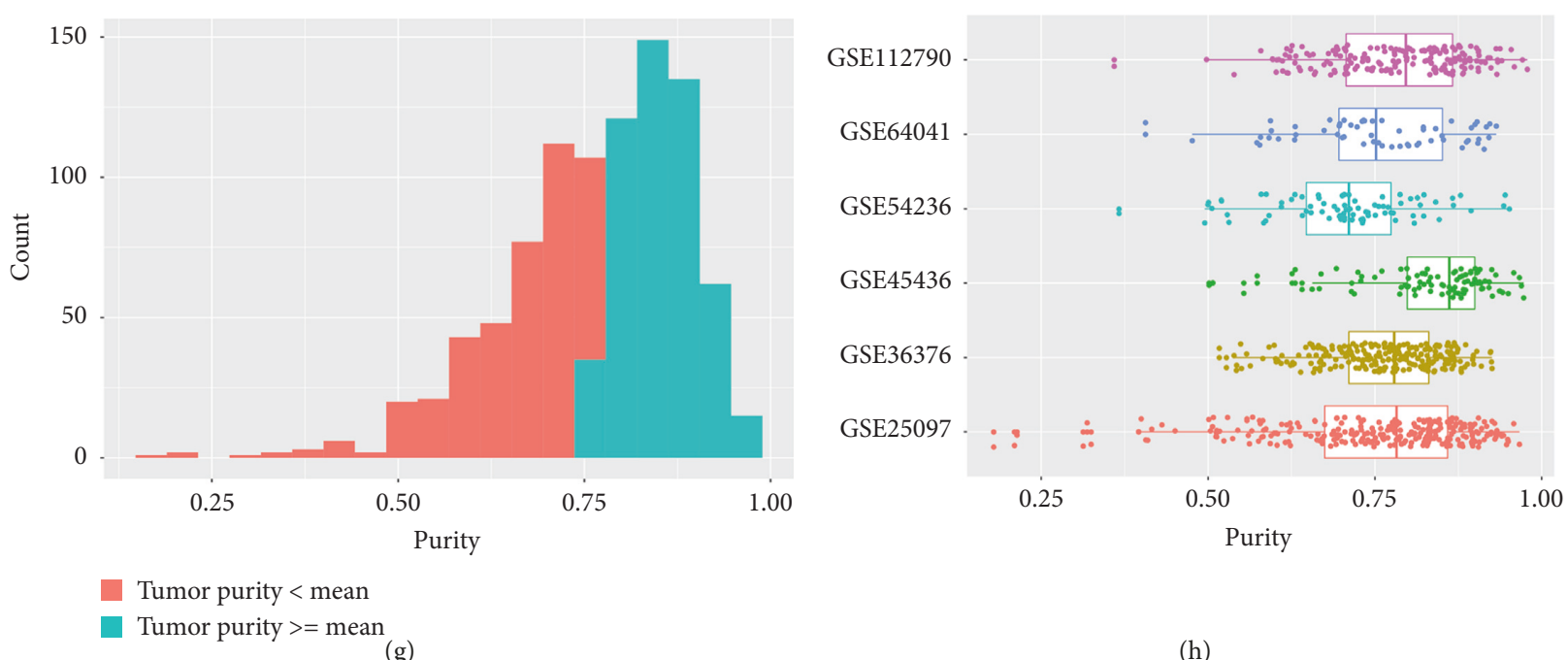

(h)

FIGURE 1: PCA of datasets and visual representation of tumor purity estimation. PCA plot of (a) GSE112790, (b) GSE64041, (c) GSE54236, (d) GSE45436, (e) GSE36376, and (f) GSE25097. (g) Histogram of tumor purity of all tumor tissues. (h) Boxplot of tumor purity in each dataset.

CCNA2 $\quad($ AUC $=0.655$, sensitivity $=0.81$, and specificity $=0.433$ ), AUPKA (AUC $=0.621$, sensitivity $=0.623$, and specificity $=0.584)$, CDKN3 (AUC $=0.613$, sensitivity $=0.941$, and specificity $=0.292)$, and $\mathrm{CDC} 20 \quad(\mathrm{AUC}=0.686$, sensitivity $=0.697$, and specificity $=0.632$ ) had certain predictive value for the 3-year survival rate of HCC patients. All of the top 20 genes in PPI exhibited a significant prognostic value (Supplementary Table S5). Then, risk score model was built based on CDC20 (coef $=0.349 ; P=2.8 e-07)$ and another gene among the top 20 genes, named NUSAP1 (coef $=-0.182 ; P=0.046)$. Compared with overall survival prediction with a single gene, the risk score model had a higher predictive value $(\mathrm{AUC}=0.71$, sensitivity $=0.789$, and specificity $=0.547)($ Figure 8$)$.

\section{Discussion}

In the present study, we had detected totally 266 DEGs. FOXM1 was the most connected gene in upregulated genes in the PPI network, which had 44 edges. Increasing evidence has suggested that FOXM1 is elevated in many tumors, such as intrahepatic cholangiocarcinoma, oesophageal adenocarcinoma, gastric cancer, cervical cancer, and HCC [18-24]. Since FOXM1 can promote the proliferation and invasion of cancer cells, it may give rise to the poor prognosis and low survival rate of patients with high FOXM1 expression [22, 24-31]. Not only that, it was also found that FOXM1 contributes to tumor angiogenesis in the study of colorectal and gastric cancer $[23,32]$. In previous studies, there was a large amount of evidence that FOXM1 directly or indirectly affects the occurrence and development of HCC, which is in line with our results $[27-29,33-39]$. In addition, in an in vivo study of HCC, the growth of tumors in mice with FOXM1 deficiency was completely stagnated, suggesting that FOXM1 has the potential to become an independent biomarker of HCC [31]. It is worth mentioning that KIF4A has been confirmed to be a downstream target of FOMX1 and the expression level of KIF4A is positively correlated with FOXM1. Overexpression of both genes will lead to excessive cell proliferation and promote tumor development [22]. Meanwhile, we observed a significant increase in the expression of KIF4A in our experimental results $(P=3.18 e-07)$, which coincided with previous studies [22]. MircoRNA plays an active role in HCC as well. For instance, the expression of microRNA-135a transcribed by FOXM1 can affect the prognosis and survival rate of patients with HCC [40]. Unfortunately, we did not build a competing endogenous RNAs (ceRNA) network in this project to find potential downstream noncoding RNAs for FOXM1, which should be investigated in future study.

FTCD was the core gene in model 2 and had a certain correlation with FOXM1. Additionally, FTCD was found useful to distinguish early HCC from benign tumors, suggesting that it might be a potential marker for HCC early diagnosis [41]. The results of enrichment analysis for FTCD were consistent with prior reports that the decrease in FTCD expression impeded the degradation pathway of histidine, which leads to the poor performance of methotrexate [42]. Therefore, we infer that patients with HCC who were not responding to methotrexate may be associated with abnormal expression of FTCD. Besides, in the available evidence, we found that autoimmune hepatitis has a $0.6 \%$ to $0.7 \%$ probability of inducing $\operatorname{HCC}[43,44]$. Interestingly, by reducing the number of circulating autoreactive T cells, the increased expression of FTCD can prevent the progression of autoimmune hepatitis [45]. Thus, low level of FTCD might contribute to high incidence of HCC and serves as a useful biomarker for primary HCC. Further research will be needed to clarify the role of FTCD in tumorigenesis.

In addition to FOXM1, CCNA2, AURKA, CDKN3, and CDC20 can be seen in the forefront of PPI. It is common knowledge that CCNA2, a core cell cycle regulator, plays a critical role with high expression from $S$ 


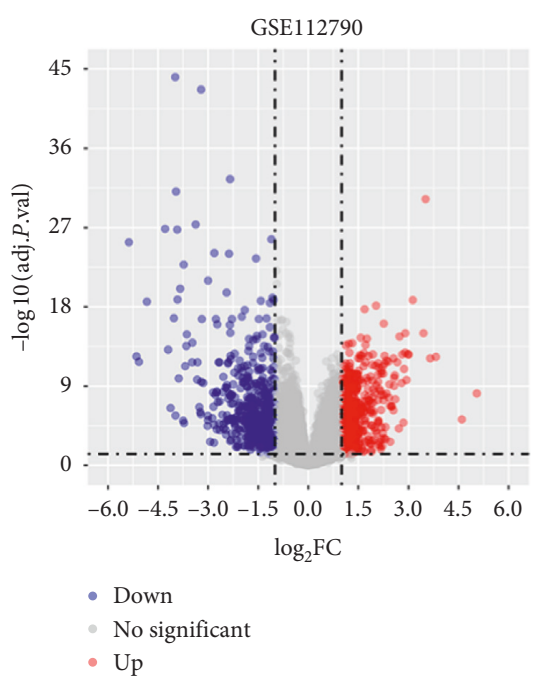

(a)

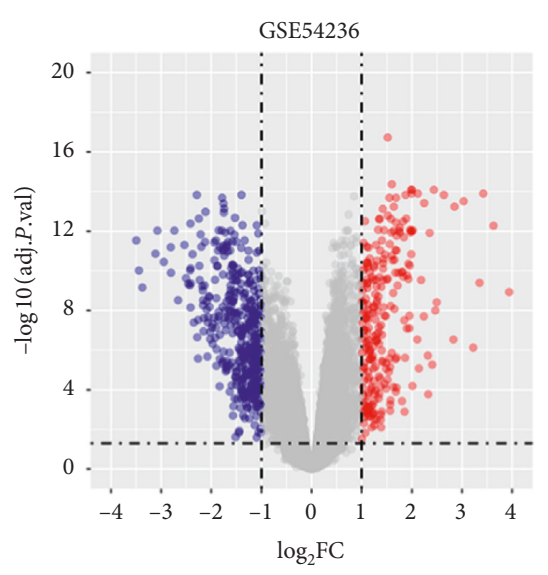

- Down

- No significant

- Up

(c)

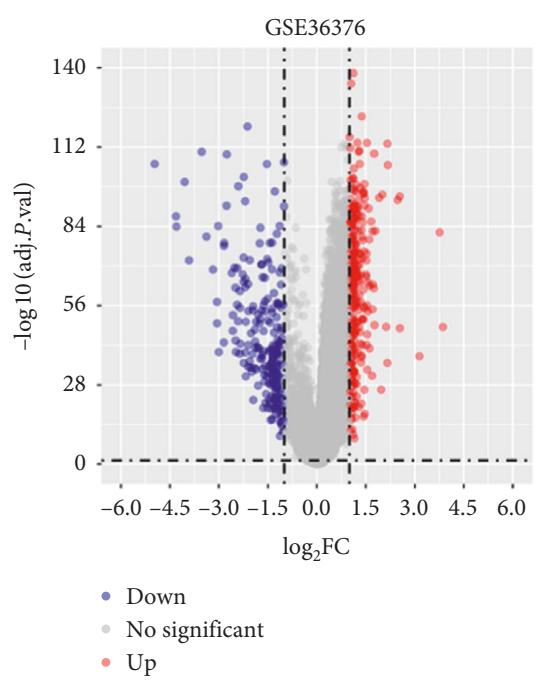

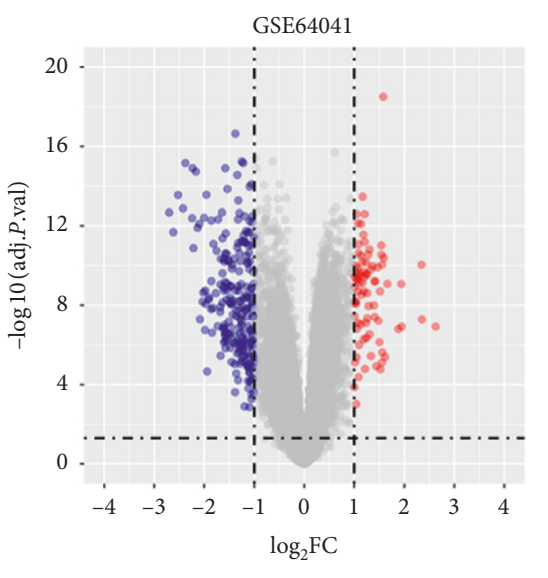

- Down

No significant

- Up

(b)

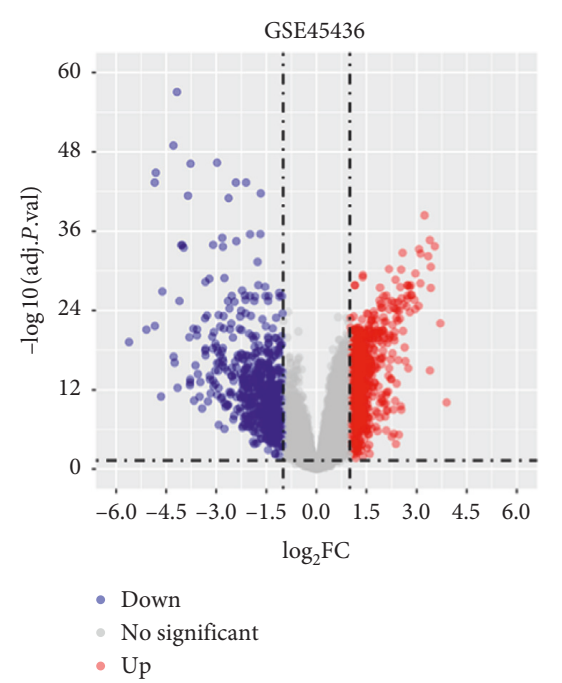

(d)

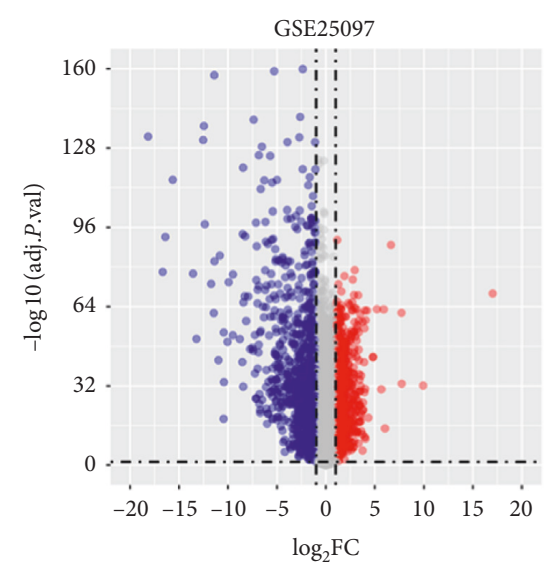

- Down

- No significant

- Up

(e)

(f)

Figure 2: Continued. 


\begin{tabular}{|c|c|c|c|c|c|c|}
\hline 4.60 & 2.35 & 3.94 & 3.90 & 3.86 & 9.91 & SPINK1 \\
\hline 2.49 & 1.95 & 2.24 & 3.71 & 3.76 & 17.03 & GPC3 \\
\hline 5.05 & 1.61 & 2.47 & 2.38 & 3.14 & 7.72 & AKR1B10 \\
\hline 1.66 & 1.14 & 1.35 & 2.17 & 1.52 & 2.65 & AURKA \\
\hline 2.86 & 1.41 & 1.97 & 3.34 & 1.52 & 1.18 & CDKN3 \\
\hline 1.75 & 1.40 & 1.27 & 1.59 & 1.56 & 4.08 & SQLE \\
\hline 2.24 & 1.54 & 1.96 & 3.11 & 2.54 & 1.58 & TOP2A \\
\hline 2.26 & 1.24 & 1.80 & 2.74 & 1.61 & 0.81 & NCAPG \\
\hline 2.02 & 1.59 & 1.98 & 2.72 & 1.59 & 0.91 & ASPM \\
\hline 1.89 & 0.54 & 2.01 & 2.31 & 1.96 & 3.21 & S100P \\
\hline 2.22 & 0.79 & 1.56 & 2.53 & 1.42 & 3.30 & PTTG1 \\
\hline 1.85 & 1.48 & 1.85 & 0.98 & 1.69 & 3.12 & LCN2 \\
\hline 2.90 & 1.28 & 1.55 & 3.39 & 1.91 & 1.32 & PRC1 \\
\hline 2.73 & 1.09 & 0.93 & 2.82 & 1.15 & 2.93 & NUSAP1 \\
\hline 3.51 & 1.57 & 1.52 & 2.39 & 2.16 & 1.30 & CAP2 \\
\hline 2.34 & 1.55 & 2.24 & 2.50 & 0.65 & 1.17 & ANLN \\
\hline 2.39 & 1.24 & 1.44 & 2.44 & 0.93 & 2.30 & UBE2T \\
\hline 2.41 & 1.19 & 1.98 & 2.70 & 0.89 & 0.60 & DLGAP5 \\
\hline 2.00 & 0.42 & 1.64 & 2.34 & 1.04 & 2.89 & UBE2C \\
\hline 3.65 & 0.60 & 1.22 & 2.66 & 1.05 & 2.33 & COL15A1 \\
\hline-4.03 & -2.62 & -2.53 & -4.81 & -3.52 & -11.39 & FCN3 \\
\hline-3.93 & -2.23 & -3.50 & -4.18 & -2.75 & -8.45 & CLEC1B \\
\hline-3.75 & -1.86 & -1.94 & -4.28 & -2.36 & -11.44 & SLC22A1 \\
\hline-4.20 & -2.02 & -1.71 & -4.62 & -4.97 & -12.45 & CYP1A2 \\
\hline-5.15 & -1.84 & -1.44 & -5.09 & -4.30 & -18.16 & HAMP \\
\hline-3.72 & -1.67 & -1.83 & -5.62 & -2.57 & -9.92 & C9 \\
\hline-4.12 & -1.94 & -2.66 & -3.79 & -2.27 & -4.33 & SLCO1B3 \\
\hline-2.28 & -1.44 & -1.64 & -2.82 & -2.57 & -7.74 & RDH16 \\
\hline-2.28 & -1.49 & -1.63 & -2.63 & -1.97 & -6.87 & SPP2 \\
\hline-2.01 & -1.52 & -1.67 & -2.45 & -2.11 & -6.67 & DNASE1L3 \\
\hline-2.87 & -1.61 & -1.61 & -3.80 & -2.25 & -5.32 & GBA3 \\
\hline-3.84 & -2.52 & -2.95 & -4.28 & -1.10 & -5.70 & OIT3 \\
\hline-2.54 & -1.14 & -1.72 & -3.02 & -2.41 & -9.48 & AFM \\
\hline-4.83 & -1.60 & -1.56 & -4.01 & -1.45 & -4.87 & CNDP1 \\
\hline-1.80 & -1.28 & -1.74 & -2.82 & -1.74 & -10.04 & MBL2 \\
\hline-2.17 & -1.10 & -1.53 & -2.37 & -2.35 & -4.70 & BHMT \\
\hline-2.74 & -1.31 & -1.49 & -3.66 & -2.25 & -5.25 & PCK1 \\
\hline-2.84 & -1.97 & -2.14 & -3.32 & -1.37 & -4.48 & $\mathrm{HAO} 2$ \\
\hline-3.88 & -1.98 & -1.44 & -4.10 & -3.02 & -6.19 & $\mathrm{APOF}$ \\
\hline-2.34 & -1.08 & -1.41 & -3.50 & -2.18 & -6.13 & CYP8B1 \\
\hline 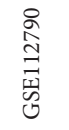 & $\begin{array}{l}\text { F्ञ } \\
\text { 岕 } \\
\text { ज्ञ } \\
\text { S }\end{array}$ & 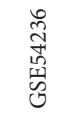 & 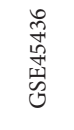 & $\begin{array}{l}\stackrel{0}{0} \\
\hat{0} \\
\widetilde{3} \\
\tilde{S} \\
0\end{array}$ & $\begin{array}{l}\hat{a} \\
\hat{O} \\
\hat{1} \\
\hat{S}\end{array}$ & \\
\hline
\end{tabular}

FIGURE 2: Volcano plot of DEGs and heatmap of the top 10 up- and downregulated genes. Volcano plot of (a) GSE112790, (b) GSE64041, (c) GSE54236, (d) GSE45436, (e) GSE36376, and (f) GSE25097. Red and blue dots in the volcano plot represent upregulated and downregulated genes, respectively, while gray represents genes that have no significant difference. (g) Heatmap of the top 10 upregulated and downregulated genes. The number in each rectangle represents the gene expression level.

phase to early mitosis $[46,47]$. It was reported that high expression of CCNA2 might induce hepatocyte nodular proliferation [48]. And the exosome circRNAs, secreted from liver adipocytes, promoted tumor growth by controlling miR-34a level and activating the USP7/CCNA2 signaling pathway [49]. All of the above findings indicate that CCNA2 directly or indirectly influences the development of HCC, which is considered as a vital part in HCC development. High expression of AURKA has previously been detected in different cancer types as well [50-52], which is implicated with the regulation of cell cycle and division [53]. With no exception to HCC, it is also described as an oncoprotein and therapeutic target. Microarray analysis pointed out that AURKA phosphorylated and stabilized hepatoma upregulated protein [54]. Moreover, a research revealed that AURKA can, in turn, give rise to malignant phenotypes of HCC by regulating HIF- $1 \alpha$ through activation of AKT and p38-MAPK signaling pathways [55]. As a result, we conjecture that it may function as a cancer-promoting gene. Excessive replication of the centrosome is considered to be a common feature of almost all human cancers [56], and 


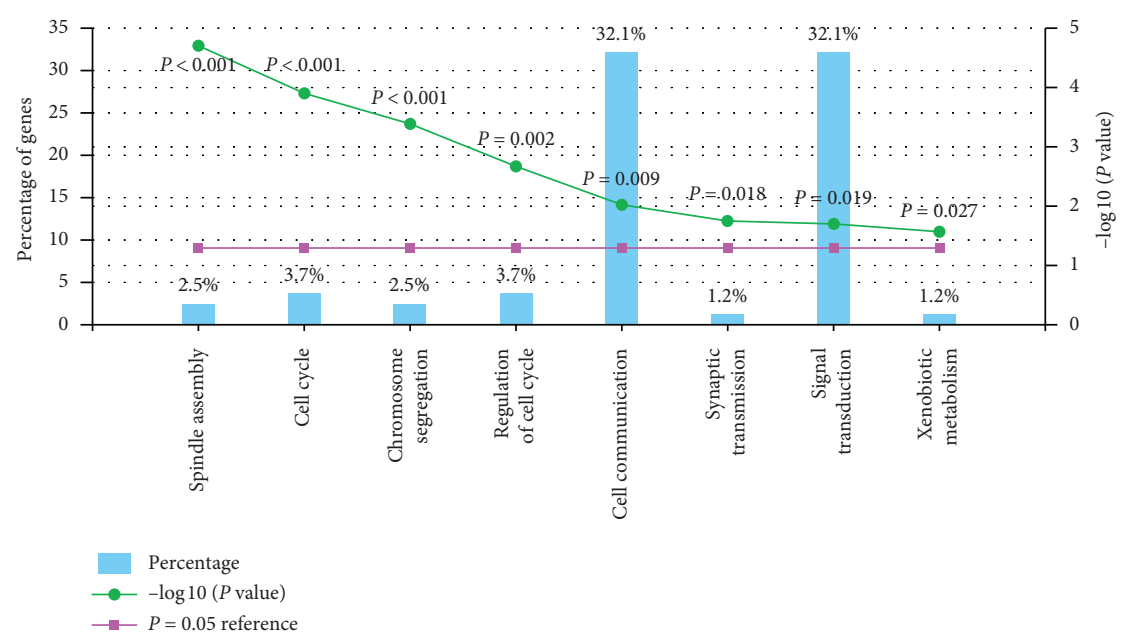

(a)

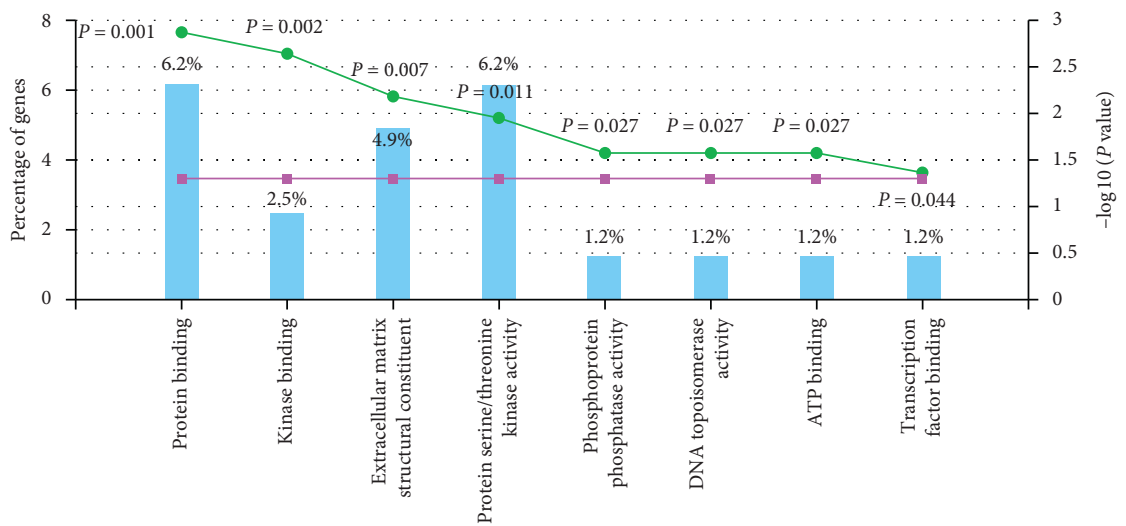

Percentage

$\longrightarrow-\log 10$ ( $P$ value)

$\longrightarrow P=0.05$ reference

(b)

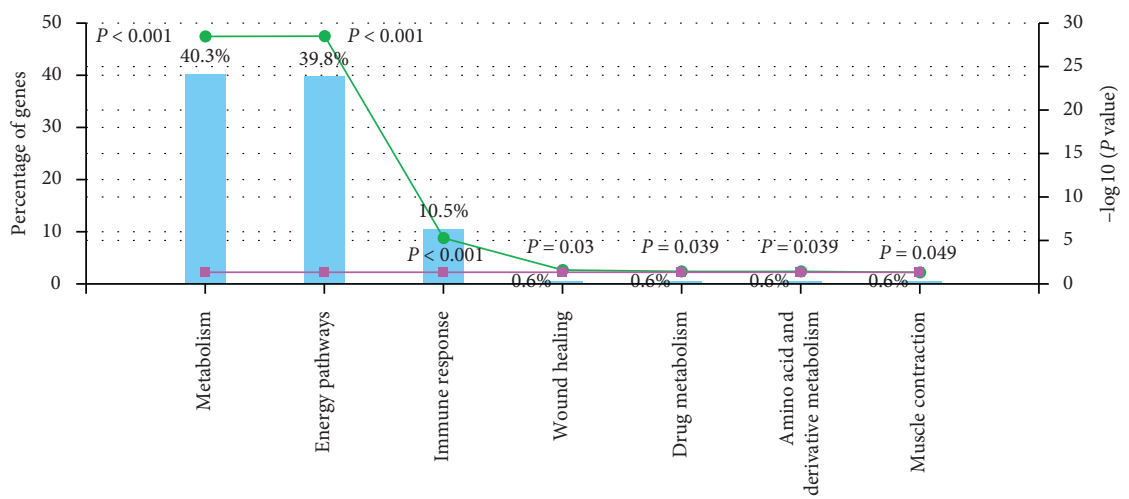

Percentage

$\longrightarrow-\log 10$ ( $P$ value)

(c)

FIgure 3: Continued. 


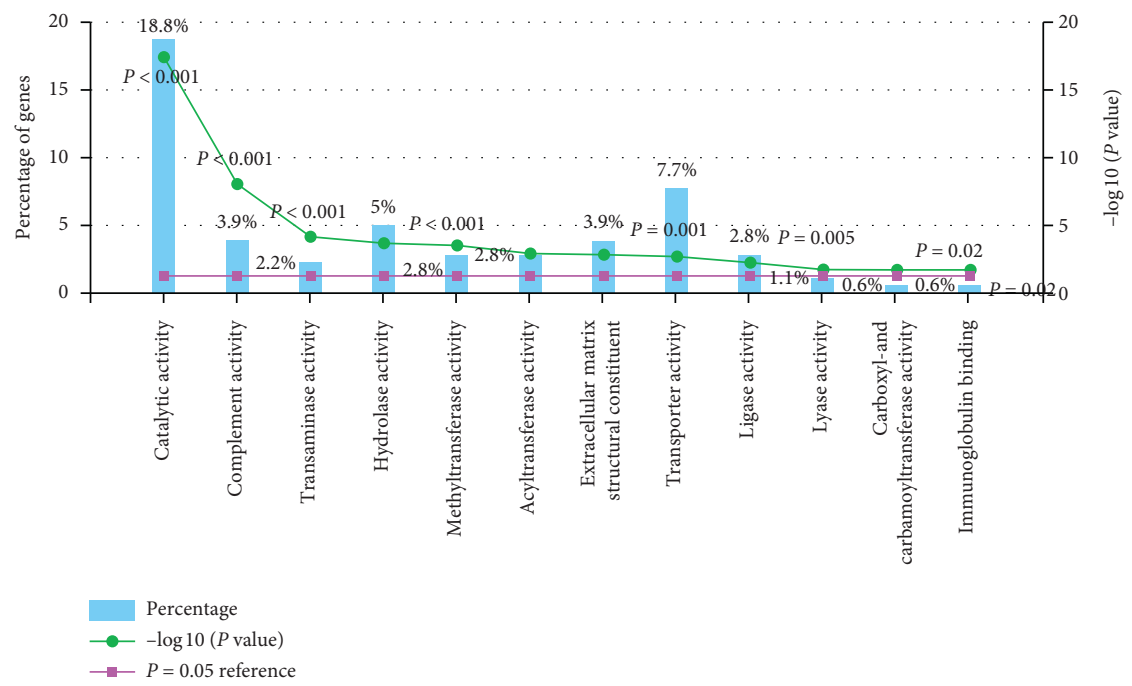

(d)

FIGURE 3: Histograms of molecular function and biological process for up- and downregulated genes. (a) Biological process for upregulated genes. (b) Molecular function for upregulated genes. (c) Biological process for downregulated genes. (d) Molecular function for downregulated genes.

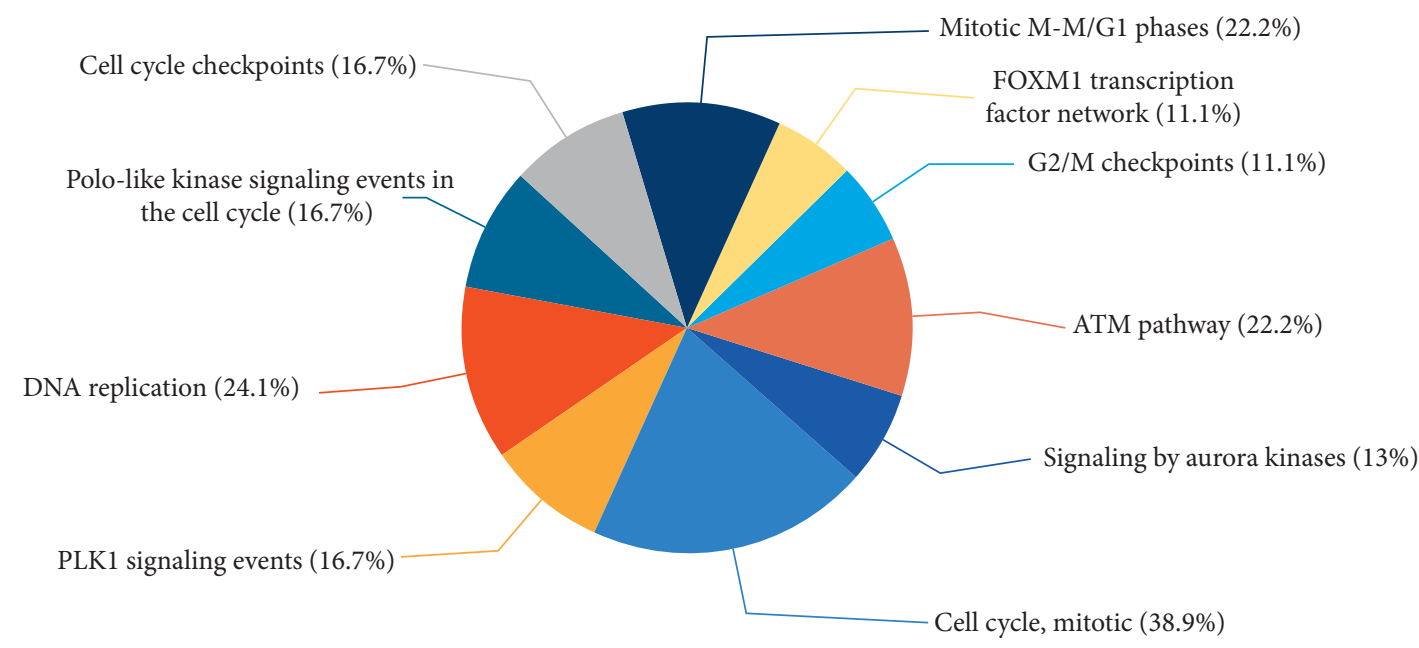

(a)

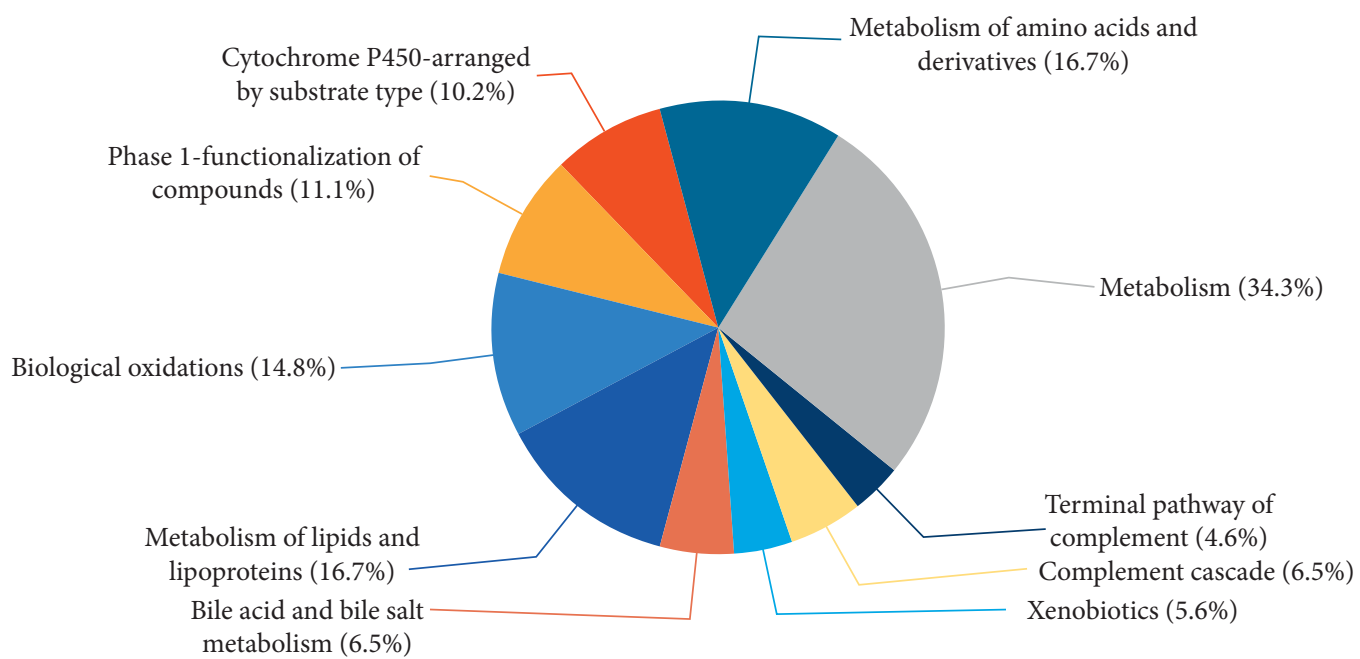

(b)

FIgURE 4: Pie charts of biological pathway for (a) upregulated genes and (b) downregulated genes. 


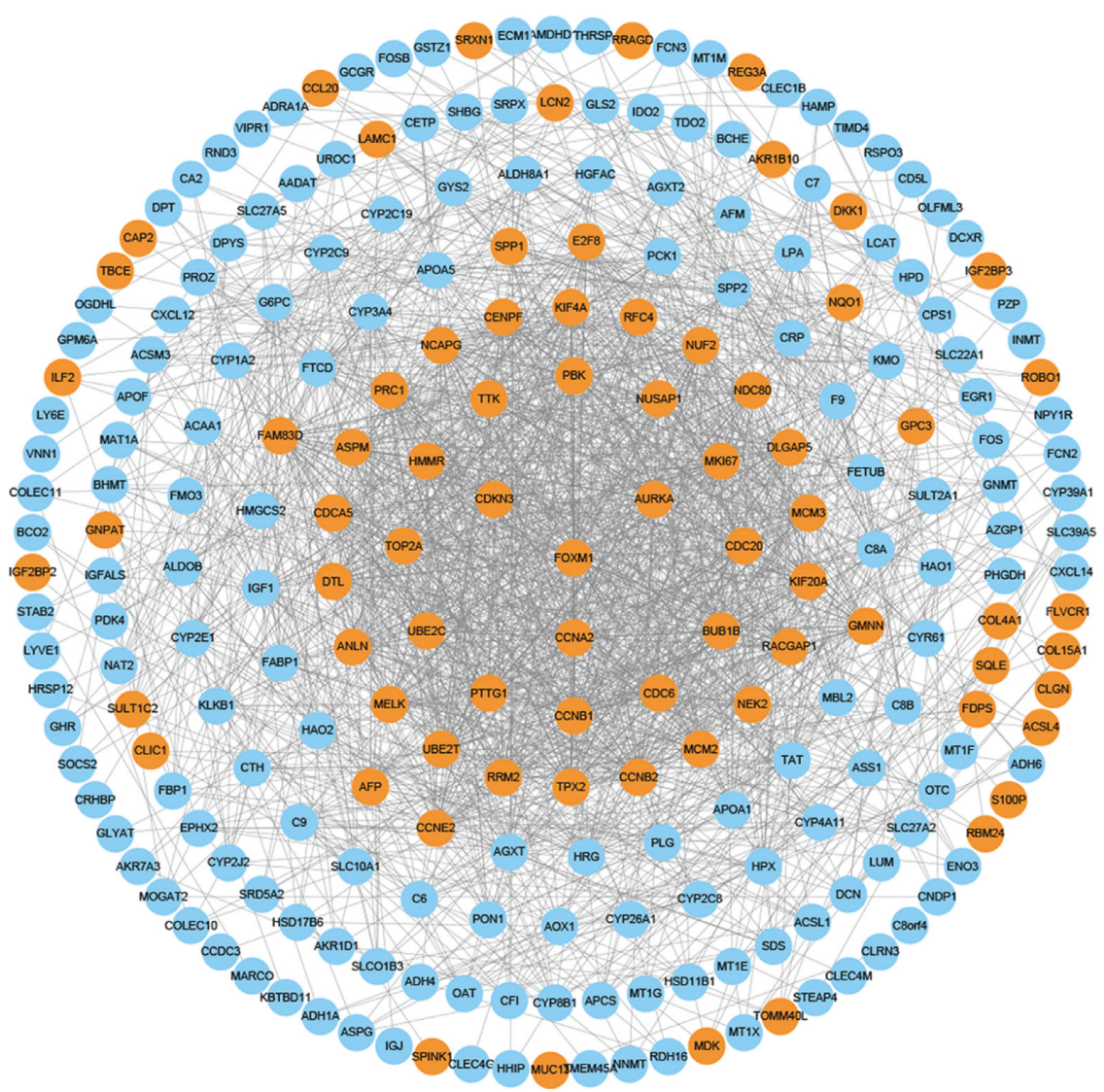

(a)

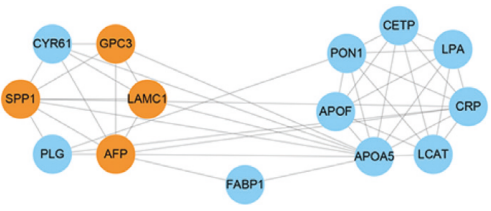

(d)

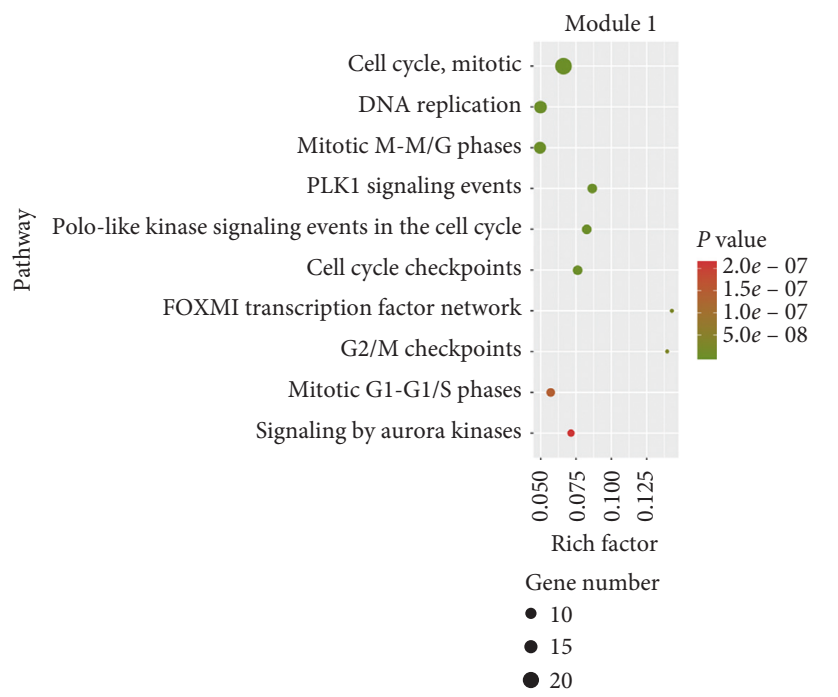

(f)

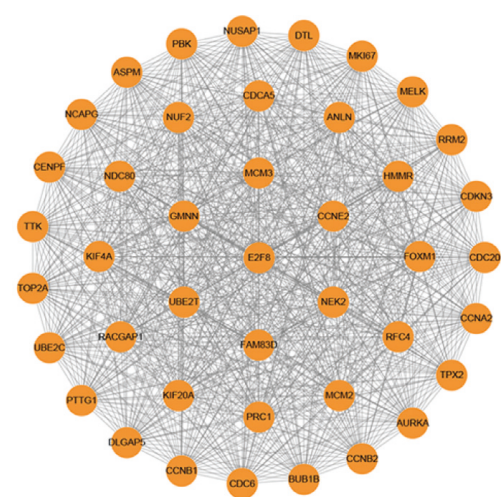

(b)

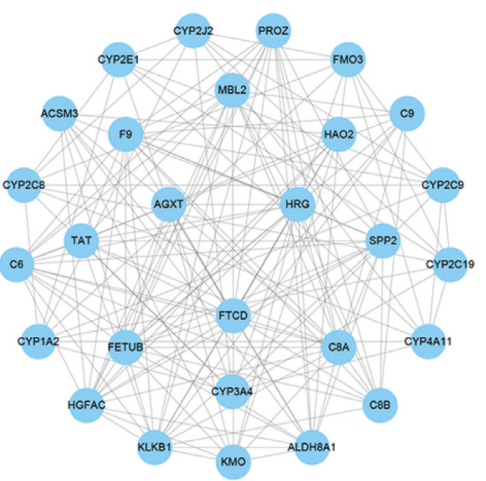

(c)

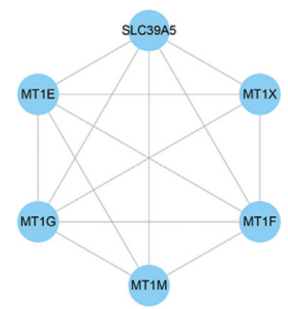

(e)

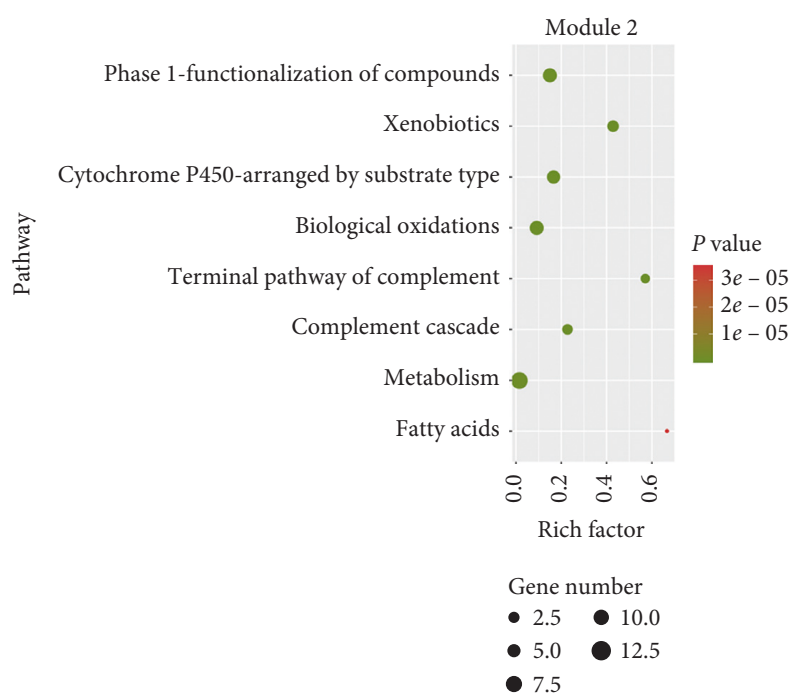

(g)

FIgURE 5: PPI network diagrams of DEGs and module network and pathway enrichment analysis of modules. (a) PPI network of DEGs. Red nodes represent upregulated genes and blue nodes represent downregulated genes. (b) Module 1, MCODE score =40.78; (c) module 2, MCODE score =11.778; (d) module 3, MCODE score =6.615; (e) module 4, MCODE score = 5.6. Pathway analysis of (f) module 1 and (g) module 2 . 


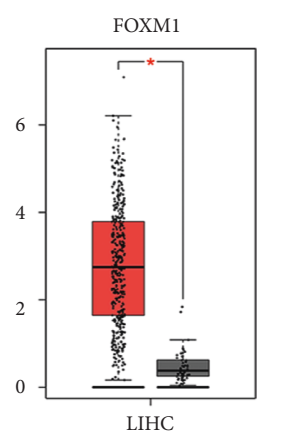

$(\operatorname{num}(T)=369 ; \operatorname{num}(N)=50)$

(a)

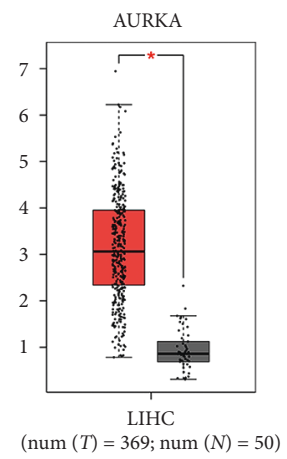

(c)

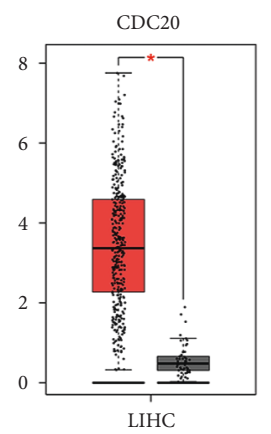

$(\operatorname{num}(T)=369 ; \operatorname{num}(N)=50)$

(e)

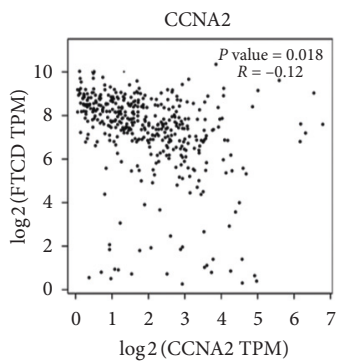

(g)

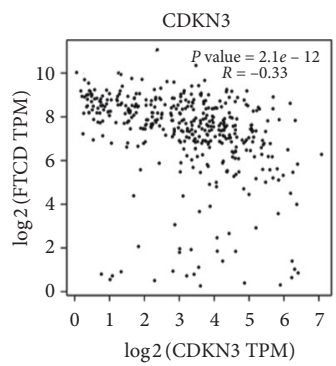

(i)

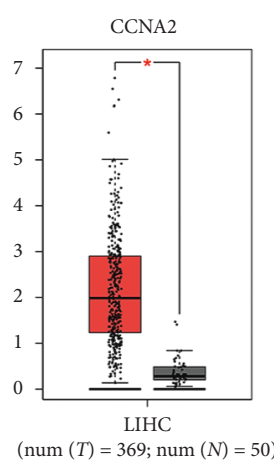

(b)

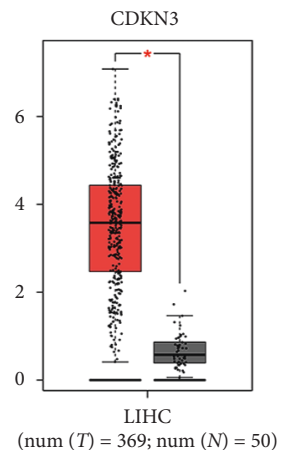

(d)

FOXM1

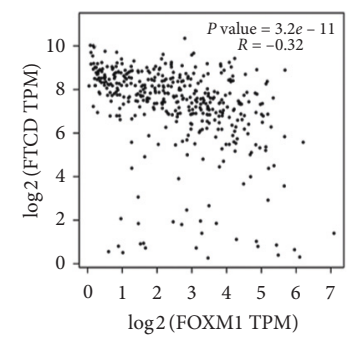

(f)

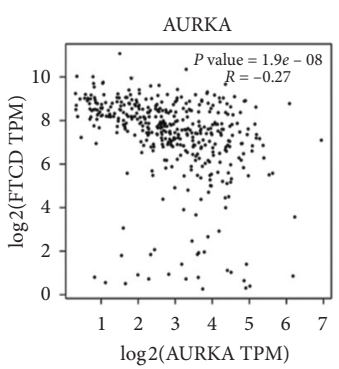

(h)

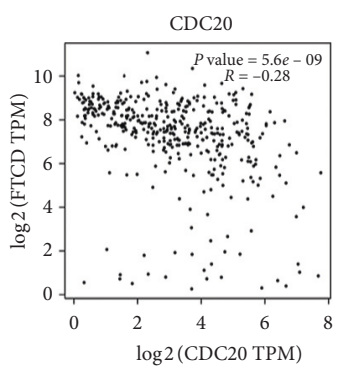

(j)

Figure 6: Continued. 


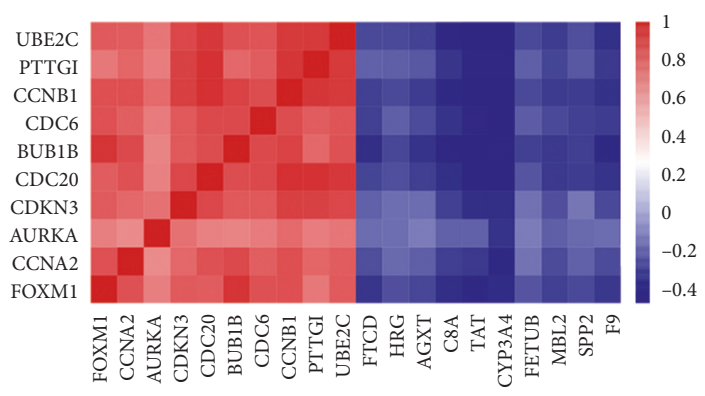

$(\mathrm{k})$

FIgURE 6: Top five hub genes expression level in liver hepatocellular carcinoma (LIHC) and correlation analysis among hub genes. (a-e) Boxplots of expression level of FOXM1, CCNA2, AURKA, CDKN3, and CDC20. (f-j) Scatter plots of correlation analysis of FTCD and the top five hub genes. (k) Heatmap of correlation coefficients among the top ten hub genes in module 1 and module 2.

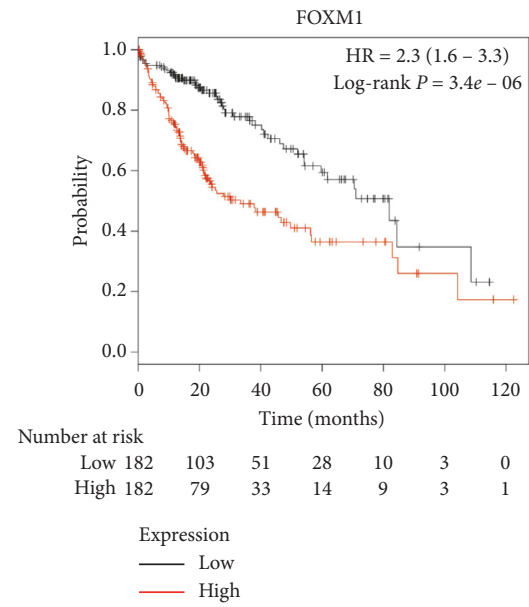

(a)

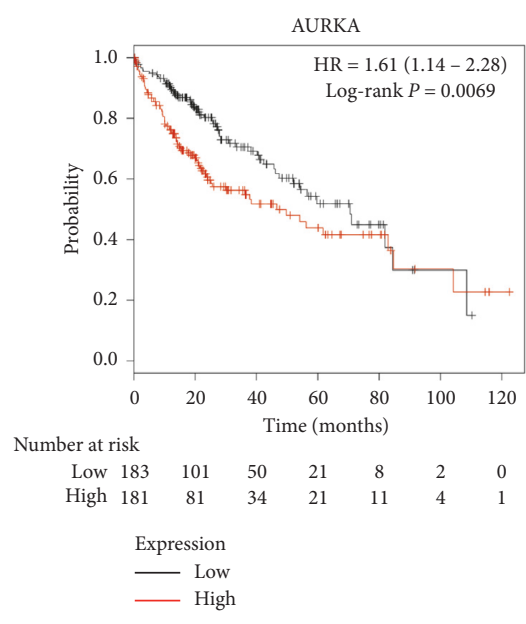

(c)

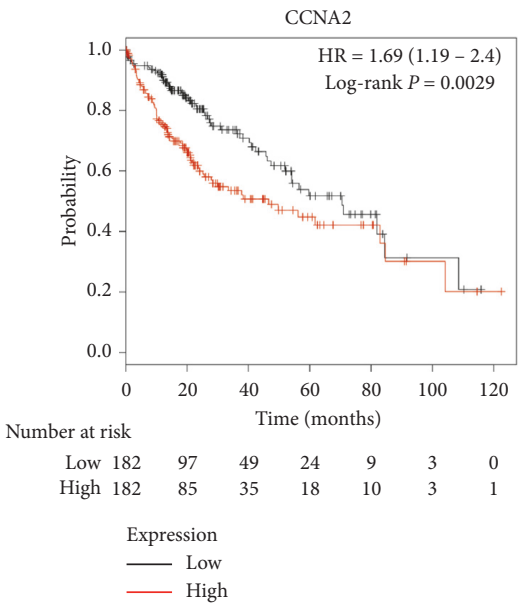

(b)

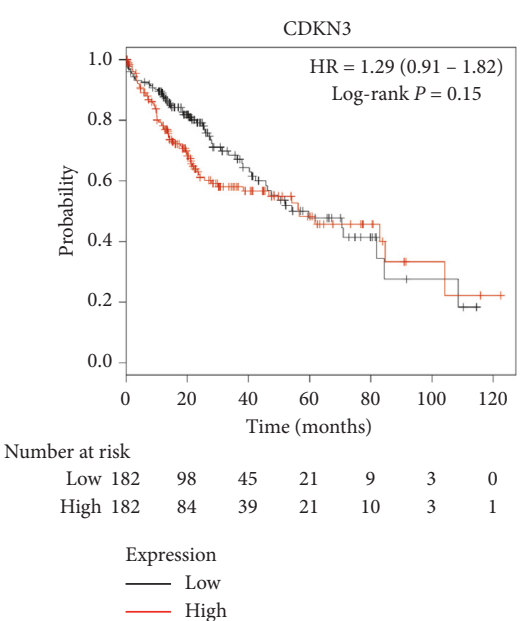

(d)

FIgure 7: Continued. 


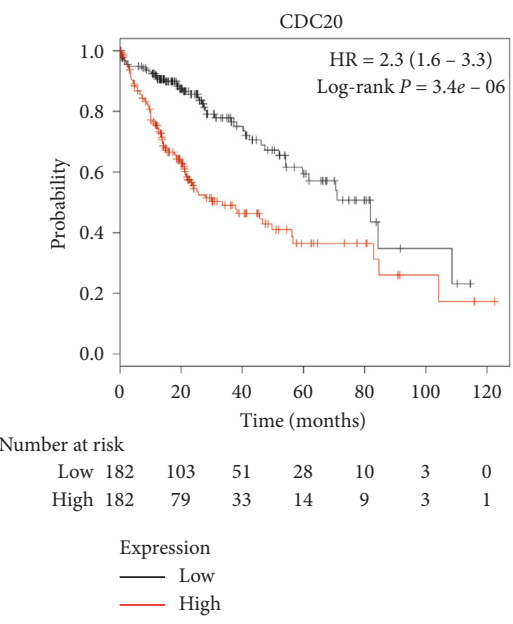

(e)

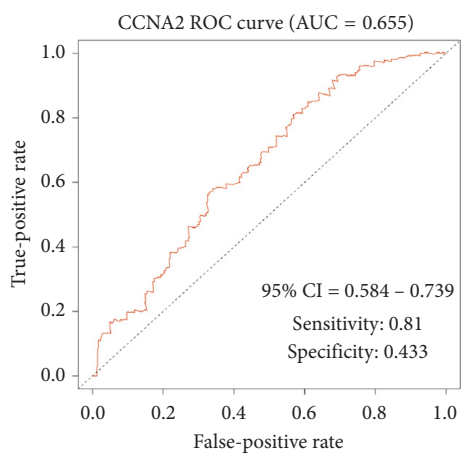

(g)

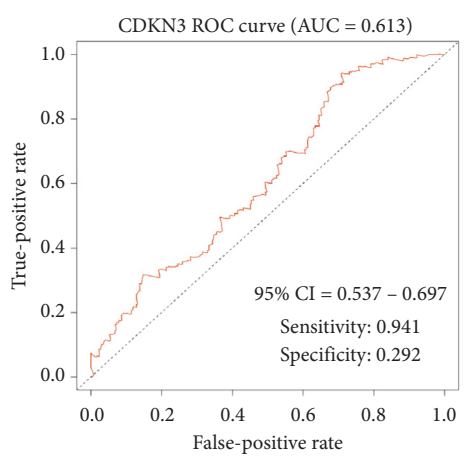

(i)

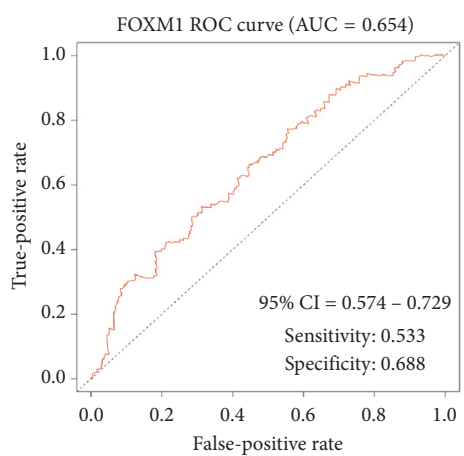

(f)

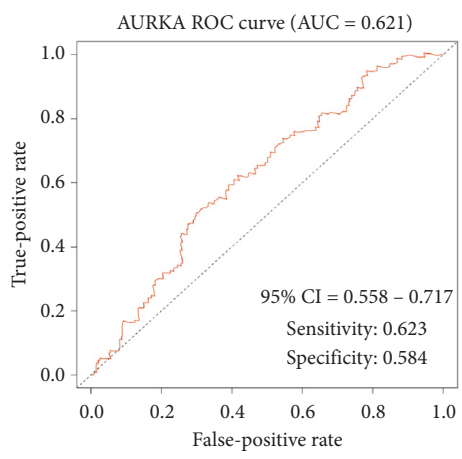

(h)

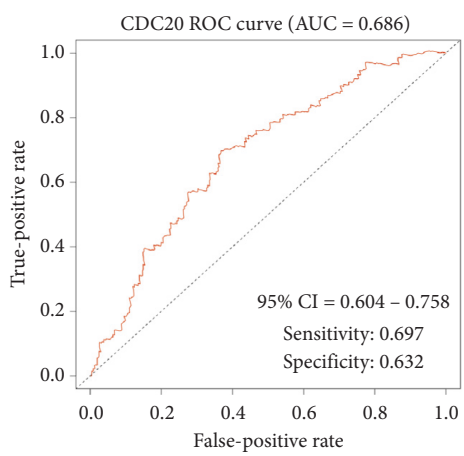

(j)

FIGURE 7: Overall survival analysis and ROC curve analysis of the top five hub genes. (a-e) Kaplan-Meier curves for FOXM1, CCNA2, AURKA, CDKN3, and CDC20. (f-j) ROC curves for predicting 3-year survival in HCC patients based on FOXM1, CCNA2, AURKA, CDKN3, and CDC20.

CDKN3 happens to have the ability to prevent this abnormality [57]. Further research indicated CDKN3 seemed to play a role in tumor suppression by CDC2 signaling pathway [58]. However, bioinformatics analysis for identification of molecular target genes in HCC revealed that the relative expression levels of CDKN3 were significantly upregulated in tumor tissues, which proved that our results were not accidental [59]. Therefore, we conjecture that the result may be due to the positive feedback regulation in the tumor microenvironment, which surely requires subsequent experiments to verify. Regrettably, since the expression level of CDKN3 is not associated with the prognosis of patients with HCC in our study, CDKN3 cannot be counted as a candidate biomarker accordingly. CDC20 played a pivotal role in the regulation of chromosome segregation and the timely end of mitosis [60]. Abnormal CDC20 expression has been detected in most human cancers [61-63], and CDC20 knockdown caused mitotic arrest to efficiently kill slippage-prone and apoptosis-resistant cancer cells [64], supporting an oncogenic role of CDC20. In conclusion, combined with literature reports and our findings, FOXM1, FTCD, CCNA2, AURKA, and CDC20 are very competitive biomarkers of HCC, while whether CDKN3 
(a)

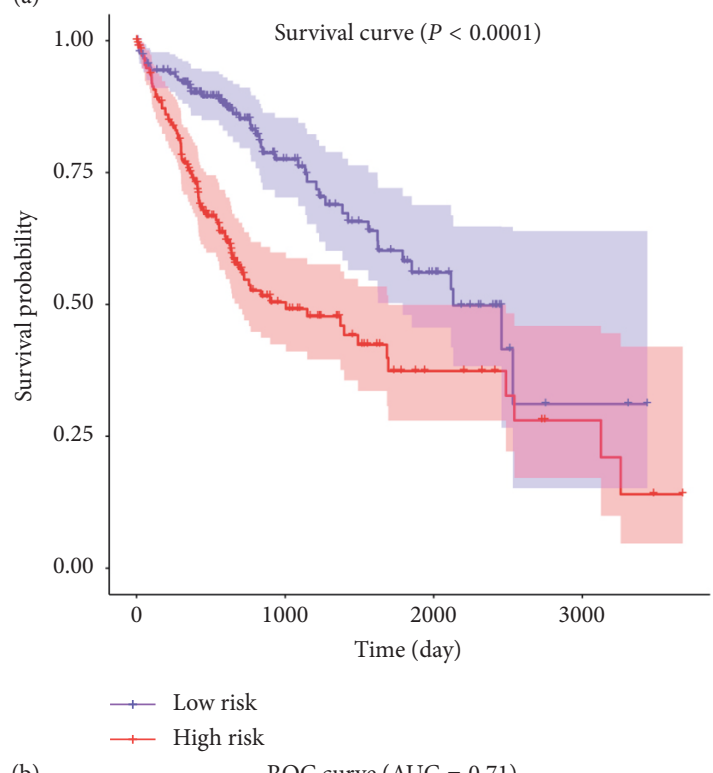

(b)

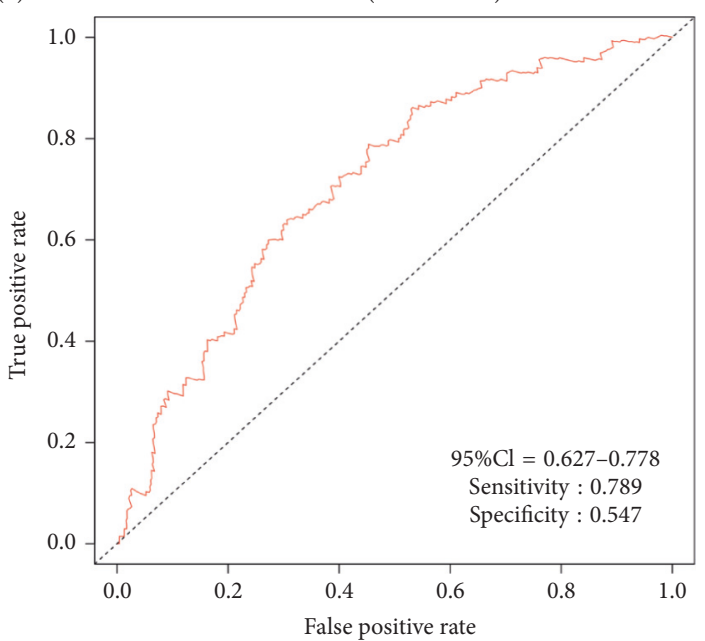

(c)
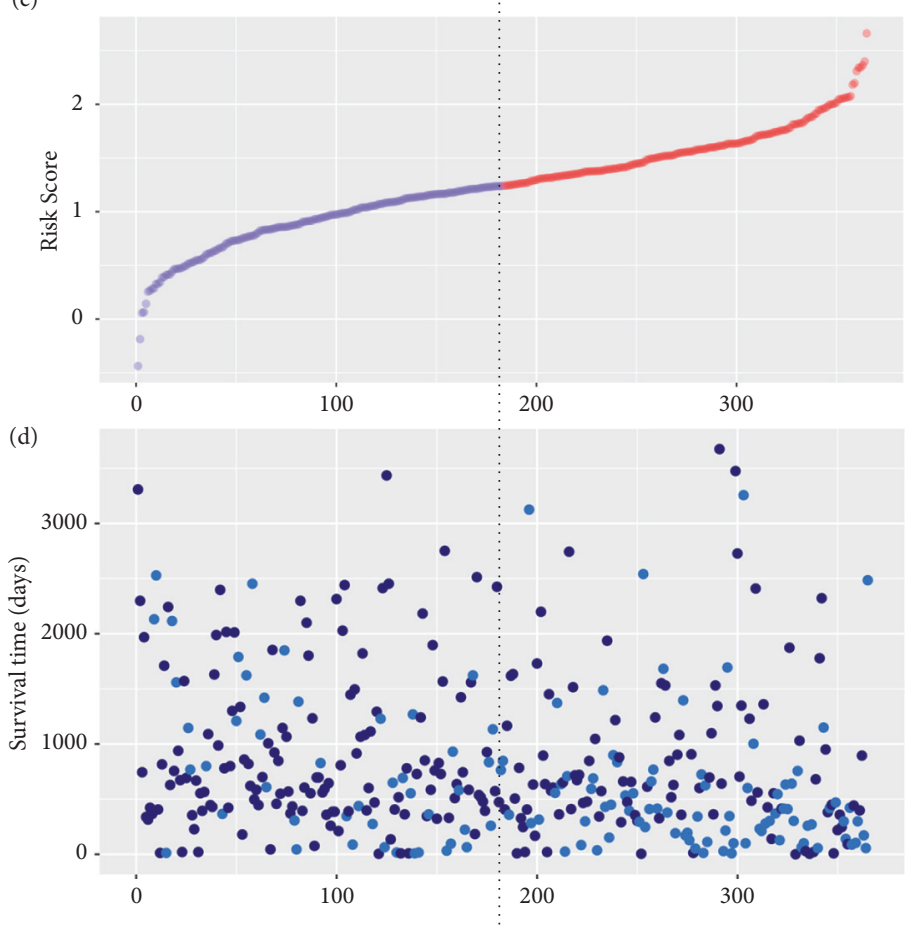

- Alive

- Dead

(e)

12

10

6

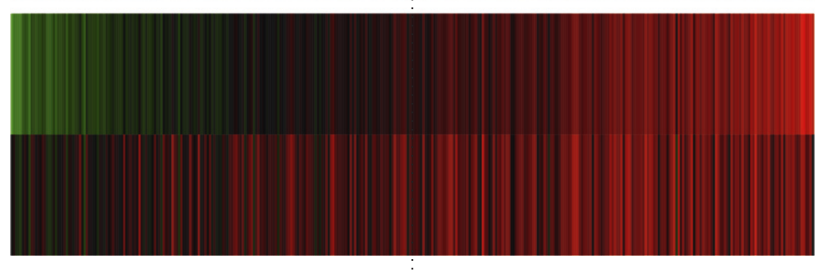

Low risk

Figure 8: Prognostic risk score model in HCC patients. (a) Kaplan-Meier curve for low- and high-risk groups. (b) ROC curve for predicting survival in HCC patients based on the risk score. (c) Volcano plot of the risk score of each individual. (d) Volcano plot of survival status and survival time of each individual. (e) Heatmap of CDC20 and NUSAP1 in low- and high-risk groups.

can be regarded as a biomarker for HCC remains further studies.

Prior to our research, there have been some reports on bioinformatics analysis of critical genes in HCC [65-68]. Nevertheless, our research still has several obvious advantages: firstly, the datasets we selected contain as many as 1557 samples and cover multiple different regions; secondly, we prioritized PCA of these datasets to ensure that tumor and normal tissues come from two distinct populations; thirdly, the method of tumor purity estimation allowed us to show readers the quality of the tumor samples in this study; fourthly, we have established a multivariate Cox proportional hazards regression model based on hub genes to improve the accuracy of single prognostic factor prediction; finally, we performed a correlation analysis between the upregulated and downregulated genes, which may reveal potential signal transduction pathways in HCC. We have to admit that our research still has the following shortcomings: first of all, our results were only based on GEO and TCGA data analysis and have not been verified; next in importance, there may be distinctions in gene expression among different types of tumors, which will be perfected in future experiments.

\section{Conclusion}

In conclusion, the integrated bioinformatics analysis was derived from 1557 tumor tissues and adjacent normal tissues in the GEO database. Tumor and normal samples came from different populations and half of the tumor samples have a purity of more than 0.765. 266 genes were eventually identified as candidate HCC biomarkers, which were enriched in signaling pathways closely related to cell proliferation and metabolic function. FOXM1, CCNA2, AURKA, CDKN3, and CDC20 were at the core of these 
genes, which opened up new horizons for diagnosis, prognosis, and treatment of HCC patients.

\section{Data Availability}

The data used to support the findings of this study are available from the corresponding author upon request.

\section{Conflicts of Interest}

No conflicts of interest exist in the submission of this manuscript.

\section{Authors' Contributions}

All authors substantially contributed to the drafting and revising of the article and the final approval of the version to be submitted. Linxin Teng contributed to the writing of the manuscript. Linxin Teng, Kaiyuan Wang, and Yu Liu contributed to data analysis, and Yanxia Ma contributed to data interpretation. Lei Bi provided research direction and manuscript revision. Weiping Chen and Lei Bi provided scientific research fund declaration and fund support. All authors read and approved the manuscript for publication.

\section{Acknowledgments}

This work was supported by grants from the National Natural Sciences Foundation of China (81873219 and 81774180), Natural Science Foundation of Jiangsu Province (BK20181425), Young Elite Scientists Sponsorship Program by CACM (CACM-2018-QNRC2-B07), and Postgraduate Research \& Practice Innovation Program of Jiangsu Province (KYCX19_1221). In addition, this work was also financially supported in part by the grants from a project funded by the Priority Academic Program Development of Jiangsu Higher Education Institutions (PAPD).

\section{Supplementary Materials}

Supplementary Table S1: information for the DEGs. Supplementary Table S2: enrichment analysis of the DEGs. Supplementary Table S3: node information for PPI network. Supplementary Table S4: pathway analysis of network modules. Supplementary Table S5: univariate Cox regression analysis of the prognosis of the top 20 genes in PPI. (Supplementary Materials)

\section{References}

[1] J. Wu, S. Yang, K. Xu et al., "Patterns and trends of liver cancer incidence rates in eastern and southeastern Asian countries (1983-2007) and predictions to 2030," Gastroenterology, vol. 154, no. 6, pp. 1719.e5-1728.e5, 2018.

[2] J. Ferlay, I. Soerjomataram, R. Dikshit et al., "Cancer incidence and mortality worldwide: sources, methods and major patterns in GLOBOCAN 2012," International Journal of Cancer, vol. 136, no. 5, pp. E359-E386, 2015.

[3] K. A. McGlynn, J. L. Petrick, and W. T. London, "Global epidemiology of hepatocellular carcinoma," Clinics in Liver Disease, vol. 19, no. 2, pp. 223-238, 2015.
[4] A. Jemal, E. M. Ward, C. J. Johnson et al., "Annual report to the nation on the status of cancer, 1975-2014, featuring survival," JNCI: Journal of the National Cancer Institute, vol. 109, no. 9, 2017.

[5] H. B. El-Serag, "Hepatocellular carcinoma," New England Journal of Medicine, vol. 365, no. 12, pp. 1118-1127, 2011.

[6] J. Bruix and M. Sherman, "Management of hepatocellular carcinoma: an update," Hepatology, vol. 53, no. 3, pp. 10201022, 2011.

[7] P.-H. Liu, C.-Y. Hsu, C.-Y. Hsia et al., "Surgical resection versus radiofrequency ablation for single hepatocellular carcinoma $\leq 2 \mathrm{~cm}$ in a propensity score model," Annals of Surgery, vol. 263, no. 3, pp. 538-545, 2016.

[8] W. K. Yun, M. S. Choi, D. Choi et al., "Superior long-term outcomes after surgery in child-pugh class a patients with single small hepatocellular carcinoma compared to radiofrequency ablation," Hepatology International, vol. 5, no. 2, pp. 722-729, 2011.

[9] M. Kaibori, K. Yoshii, I. Yokota et al., "Impact of advanced age on survival in patients undergoing resection of hepatocellular carcinoma," Annals of Surgery, vol. 269, no. 4, pp. 692-699, 2019.

[10] J. M. Llovet, S. Ricci, V. Mazzaferro et al., "Sorafenib in advanced hepatocellular carcinoma," New England Journal of Medicine, vol. 359, no. 4, pp. 378-390, 2008.

[11] A. B. Siegel, S. K. Olsen, A. Magun, and R. S. Brown Jr., "Sorafenib: where do we go from here?," Hepatology, vol. 52, no. 1, pp. 360-369, 2010.

[12] R. Rudalska, D. Dauch, T. Longerich et al., "In vivo RNAi screening identifies a mechanism of sorafenib resistance in liver cancer," Nature Medicine, vol. 20, no. 10, pp. 1138-1146, 2014.

[13] M. Pathan, S. Keerthikumar, D. Chisanga et al., "A novel community driven software for functional enrichment analysis of extracellular vesicles data," Journal of Extracellular Vesicles, vol. 6, no. 1, Article ID 1321455, 2017.

[14] D. Szklarczyk, A. L. Gable, D. Lyon et al., "STRING v11: protein-protein association networks with increased coverage, supporting functional discovery in genome-wide experimental datasets," Nucleic Acids Research, vol. 47, no. D1, pp. D607-d613, 2019.

[15] D. Otasek, J. H. Morris, J. Boucas, A. R. Pico, and B. Demchak, "Cytoscape automation: empowering workflow-based network analysis," Genome Biology, vol. 20, no. 1, p. 185, 2019.

[16] Z. Tang, C. Li, B. Kang, G. Gao, C. Li, and Z. Zhang, "GEPIA: a web server for cancer and normal gene expression profiling and interactive analyses," Nucleic Acids Research, vol. 45, no. W1, pp. W98-W102, 2017.

[17] A. Nagy, A. Lánczky, O. Menyhárt, and B. Győrffy, "Validation of miRNA prognostic power in hepatocellular carcinoma using expression data of independent datasets," Scientific Reports, vol. 8, no. 1, p. 9227, 2018.

[18] L. Liu, J. Wu, Y. Guo et al., "Overexpression of FoxM1 predicts poor prognosis of intrahepatic cholangiocarcinoma," Aging, vol. 10, no. 12, pp. 4120-4140, 2018.

[19] M. Dibb, N. Han, J. Choudhury et al., "The FOXM1-PLK1 axis is commonly upregulated in oesophageal adenocarcinoma," British Journal of Cancer, vol. 107, no. 10, pp. 1766-1775, 2012.

[20] J. Zeng, L. Wang, Q. Li et al., "FoxM1 is up-regulated in gastric cancer and its inhibition leads to cellular senescence, partially dependent on p27kip1," The Journal of Pathology, vol. 218, no. 4, pp. 419-427, 2009.

[21] D. Chan, S. Yu, P. Chiu et al., "Over-expression of FOXM1 transcription factor is associated with cervical cancer 
progression and pathogenesis," The Journal of Pathology, vol. 215, no. 3, pp. 245-252, 2008.

[22] G. Hu, Z. Yan, C. Zhang et al., "FOXM1 promotes hepatocellular carcinoma progression by regulating KIF4A expression," Journal of Experimental \& Clinical Cancer Research, vol. 38, no. 1, p. 188, 2019.

[23] Y. Wang, M. Wu, Z. Lei et al., "Dysregulation of miR-68685p/FOXM1 circuit contributes to colorectal cancer angiogenesis," Journal of Experimental \& Clinical Cancer Research, vol. 37, no. 1, p. 292, 2018.

[24] X. Zhang, Q. L. Lv, Y. T. Huang, L. H. Zhang, and H. H. Zhou, "Akt/FoxM1 signaling pathway-mediated upregulation of MYBL2 promotes progression of human glioma," Journal of Experimental \& Clinical Cancer Research, vol. 36, no. 1, p. 105, 2017.

[25] C. Bai, X. Liu, C. Qiu, and J. Zheng, "FoxM1 is regulated by both HIF- $1 \alpha$ and HIF- $2 \alpha$ and contributes to gastrointestinal stromal tumor progression," Gastric Cancer, vol. 22, no. 1, pp. 91-103, 2019.

[26] A. Smirnov, E. Panatta, A. Lena et al., "FOXM1 regulates proliferation, senescence and oxidative stress in keratinocytes and cancer cells," Aging, vol. 8, no. 7, pp. 1384-1397, 2016.

[27] C. Tian, H. Wu, C. Li et al., "Downreguation of FoxM1 by miR-214 inhibits proliferation and migration in hepatocellular carcinoma," Gene Therapy, vol. 25, no. 4, pp. 312-319, 2018.

[28] F.-D. Meng, J. C. Wei, K. Qu et al., "FoxM1 overexpression promotes epithelial-mesenchymal transition and metastasis of hepatocellular carcinoma," World Journal of Gastroenterology, vol. 21, no. 1, pp. 196-213, 2015.

[29] L. Xia, W. Huang, D. Tian et al., "Upregulated FoxM1 expression induced by hepatitis $\mathrm{B}$ virus $\mathrm{X}$ protein promotes tumor metastasis and indicates poor prognosis in hepatitis $\mathrm{B}$ virus-related hepatocellular carcinoma," Journal of Hepatology, vol. 57, no. 3, pp. 600-612, 2012.

[30] H. J. Park, G. Gusarova, Z. Wang et al., "Deregulation of FoxM1b leads to tumour metastasis," EMBO Molecular Medicine, vol. 3, no. 1, pp. 21-34, 2011.

[31] V. V. Kalinichenko, M. L. Major, X. Wang et al., "Foxm1b transcription factor is essential for development of hepatocellular carcinomas and is negatively regulated by the p19ARF tumor suppressor," Genes \& Development, vol. 18, no. 7, pp. 830-850, 2004.

[32] L. Wang, X. Bo, Q. Zheng, W. Ge, Y. Liu, and B. Li, "Paired box 8 suppresses tumor angiogenesis and metastasis in gastric cancer through repression of FOXM1 via induction of microRNA-612," Journal of Experimental \& Clinical Cancer Research, vol. 37, no. 1, p. 159, 2018.

[33] V. Chand, A. Pandey, D. Kopanja, G. Guzman, and P. Raychaudhuri, "Opposing roles of the forkhead box factors FoxM1 and FoxA2 in liver cancer," Molecular Cancer Research, vol. 17, no. 5, pp. 1063-1074, 2019.

[34] M. Han, H. Lu, K. Han et al., "XTP8 promotes hepatocellular carcinoma growth by forming a positive feedback loop with FOXM1 oncogene," Biochemical and Biophysical Research Communications, vol. 515, no. 3, pp. 455-461, 2019.

[35] N. Chai, H.-H. Xie, J.-P. Yin et al., "FOXM1 promotes proliferation in human hepatocellular carcinoma cells by transcriptional activation of CCNB1," Biochemical and Biophysical Research Communications, vol. 500, no. 4, pp. 924929, 2018.

[36] R. Shang, M. Pu, Y. Li, and D. Wang, "FOXM1 regulates glycolysis in hepatocellular carcinoma by transactivating glucose transporter 1 expression," Oncology Reports, vol. 37, no. 4, pp. 2261-2269, 2017.

[37] D. Kopanja, A. Pandey, M. Kiefer et al., "Essential roles of FoxM1 in Ras-induced liver cancer progression and in cancer cells with stem cell features," Journal of Hepatology, vol. 63, no. 2, pp. 429-436, 2015.

[38] C. Ho, C. Wang, S. Mattu et al., “AKT (v-akt murine thymoma viral oncogene homolog 1) and N-Ras (neuroblastoma ras viral oncogene homolog) coactivation in the mouse liver promotes rapid carcinogenesis by way of mTOR (mammalian target of rapamycin complex 1), FOXM1 (forkhead box M1)/ SKP2, and c-Myc pathways," Hepatology, vol. 55, no. 3, pp. 833-845, 2012.

[39] T. Yamashita, A. Kitao, O. Matsui et al., "Gd-EOB-DTPAenhanced magnetic resonance imaging and alpha-fetoprotein predict prognosis of early-stage hepatocellular carcinoma," Hepatology, vol. 60, no. 5, pp. 1674-1685, 2014.

[40] S. Liu, W. Guo, J. Shi et al., "MicroRNA-135a contributes to the development of portal vein tumor thrombus by promoting metastasis in hepatocellular carcinoma," Journal of Hepatology, vol. 56, no. 2, pp. 389-396, 2012.

[41] M. Seimiya, T. Tomonaga, K. Matsushita et al., "Identification of novel immunohistochemical tumor markers for primary hepatocellular carcinoma; clathrin heavy chain and formiminotransferase cyclodeaminase," Hepatology, vol. 48, no. 2, pp. 519-530, 2008.

[42] N. Kanarek, H. R. Keys, J. R. Cantor et al., "Histidine catabolism is a major determinant of methotrexate sensitivity," Nature, vol. 559, no. 7715, pp. 632-636, 2018.

[43] L. Grønbæk, H. Vilstrup, and P. Jepsen, "Autoimmune hepatitis in Denmark: incidence, prevalence, prognosis, and causes of death. A nationwide registry-based cohort study," Journal of Hepatology, vol. 60, no. 3, pp. 612-617, 2014.

[44] A. D. Yeoman, T. Al-Chalabi, J. B. Karani et al., "Evaluation of risk factors in the development of hepatocellular carcinoma in autoimmune hepatitis: implications for follow-up and screening," Hepatology, vol. 48, no. 3, pp. 863-870, 2008.

[45] P. Lapierre, K. Béland, R. Yang, and F. Alvarez, "Adoptive transfer of ex vivo expanded regulatory $\mathrm{T}$ cells in an autoimmune hepatitis murine model restores peripheral tolerance," Hepatology, vol. 57, no. 1, pp. 217-227, 2013.

[46] K. Baumann, "Genome stability: cyclin' on mRNA," Nature Reviews Molecular Cell Biology, vol. 17, no. 11, pp. 676-677, 2016.

[47] H. Hochegger, S. Takeda, and T. Hunt, "Cyclin-dependent kinases and cell-cycle transitions: does one fit all?," Nature Reviews Molecular Cell Biology, vol. 9, no. 11, pp. 910-916, 2008.

[48] H.-C. Wang, W.-T. Chang, W.-W. Chang et al., "Hepatitis B virus pre-S2 mutant upregulates cyclin A expression and induces nodular proliferation of hepatocytes," Hepatology, vol. 41, no. 4, pp. 761-770, 2005.

[49] H. Zhang, T. Deng, S. Ge et al., "Exosome circRNA secreted from adipocytes promotes the growth of hepatocellular carcinoma by targeting deubiquitination-related USP7," Oncogene, vol. 38, no. 15, pp. 2844-2859, 2019.

[50] Y. Xie, S. Zhu, M. Zhong et al., "Inhibition of aurora kinase a induces necroptosis in pancreatic carcinoma," Gastroenterology, vol. 153, no. 5, pp. 1429.e5-1443.e5, 2017.

[51] L. Wang-Bishop, Z. Chen, A. Gomaa et al., "Inhibition of AURKA reduces proliferation and survival of gastrointestinal cancer cells with activated KRAS by preventing activation of RPS6KB1," Gastroenterology, vol. 156, no. 3, pp. 662.e7675.e7, 2019. 
[52] A. Katsha, M. Soutto, V. Sehdev et al., "Aurora kinase A promotes inflammation and tumorigenesis in mice and human gastric neoplasia," Gastroenterology, vol. 145, no. 6, pp. 1312.e8-1322.e8, 2013.

[53] M. Carmena and W. C. Earnshaw, "The cellular geography of aurora kinases," Nature Reviews Molecular Cell Biology, vol. 4, no. 11, pp. 842-854, 2003.

[54] C.-T. R. Yu, J.-M. Hsu, Y.-C. G. Lee, A.-P. Tsou, C.-K. Chou, and C.-Y. F. Huang, "Phosphorylation and stabilization of HURP by Aurora-A: implication of HURP as a transforming target of Aurora-A," Molecular and Cellular Biology, vol. 25, no. 14, pp. 5789-5800, 2005.

[55] S.-Y. Cui, J.-Y. Huang, Y.-T. Chen et al., "The role of Aurora A in hypoxia-inducible factor $1 \alpha$-promoting malignant phenotypes of hepatocelluar carcinoma," Cell Cycle, vol. 12, no. 17, pp. 2849-2866, 2013.

[56] S. A. Godinho and D. Pellman, "Causes and consequences of centrosome abnormalities in cancer," Philosophical Transactions of the Royal Society B: Biological Sciences, vol. 369, no. 1650, Article ID 20130467, 2014.

[57] V. Srinivas, M. Kitagawa, J. Wong, P.-J. Liao, and S. H. Lee, "The tumor suppressor Cdkn3 is required for maintaining the proper number of centrosomes by regulating the centrosomal stability of Mps1," Cell Reports, vol. 13, no. 8, pp. 1569-1577, 2015.

[58] G. Nalepa, J. Barnholtz-Sloan, R. Enzor et al., "The tumor suppressor CDKN3 controls mitosis," The Journal of Cell Biology, vol. 201, no. 7, pp. 997-1012, 2013.

[59] L. Zhou, Y. Du, L. Kong, X. Zhang, and Q. Chen, "Identification of molecular target genes and key pathways in hepatocellular carcinoma by bioinformatics analysis," OncoTargets and Therapy, vol. 2018, no. 11, pp. 1861-1869, 2018.

[60] M. Kapanidou, N. L. Curtis, and V. M. Bolanos-Garcia, "Cdc20: at the crossroads between chromosome segregation and mitotic exit," Trends in Biochemical Sciences, vol. 42, no. 3, pp. 193-205, 2017.

[61] J.-W. Choi, Y. Kim, J.-H. Lee, and Y.-S. Kim, "High expression of spindle assembly checkpoint proteins CDC20 and MAD2 is associated with poor prognosis in urothelial bladder cancer," Virchows Archiv, vol. 463, no. 5, pp. 681-687, 2013.

[62] Y. Kim, J.-W. Choi, J.-H. Lee, and Y.-S. Kim, "Spindle assembly checkpoint MAD2 and CDC20 overexpressions and cell-in-cell formation in gastric cancer and its precursor lesions," Human Pathology, vol. 85, pp. 174-183, 2019.

[63] J. Li, J.-Z. Gao, J.-L. Du, Z.-X. Huang, and L.-X. Wei, "Increased CDC20 expression is associated with development and progression of hepatocellular carcinoma," International Journal of Oncology, vol. 45, no. 4, pp. 1547-1555, 2014.

[64] H.-C. Huang, J. Shi, J. D. Orth, and T. J. Mitchison, "Evidence that mitotic exit is a better cancer therapeutic target than spindle assembly," Cancer Cell, vol. 16, no. 4, pp. 347-358, 2009.

[65] L. Li, Q. Lei, S. Zhang, L. Kong, and B. Qin, "Screening and identification of key biomarkers in hepatocellular carcinoma: evidence from bioinformatic analysis," Oncology Reports, vol. 38, no. 5, pp. 2607-2618, 2017.

[66] J. Long, Y. Bai, X. Yang et al., "Construction and comprehensive analysis of a ceRNA network to reveal potential prognostic biomarkers for hepatocellular carcinoma," Cancer Cell International, vol. 19, no. 1, p. 90, 2019.

[67] S. Shen, J. Kong, Y. Qiu, X. Yang, W. Wang, and L. Yan, "Identification of core genes and outcomes in hepatocellular carcinoma by bioinformatics analysis," Journal of Cellular Biochemistry, vol. 120, no. 6, pp. 10069-10081, 2019.

[68] M. Ni, X. Liu, J. Wu et al., "Identification of candidate biomarkers correlated with the pathogenesis and prognosis of non-small cell lung cancer via integrated bioinformatics analysis," Frontiers in Genetics, vol. 9, p. 469, 2018. 


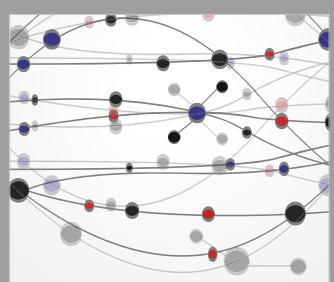

The Scientific World Journal
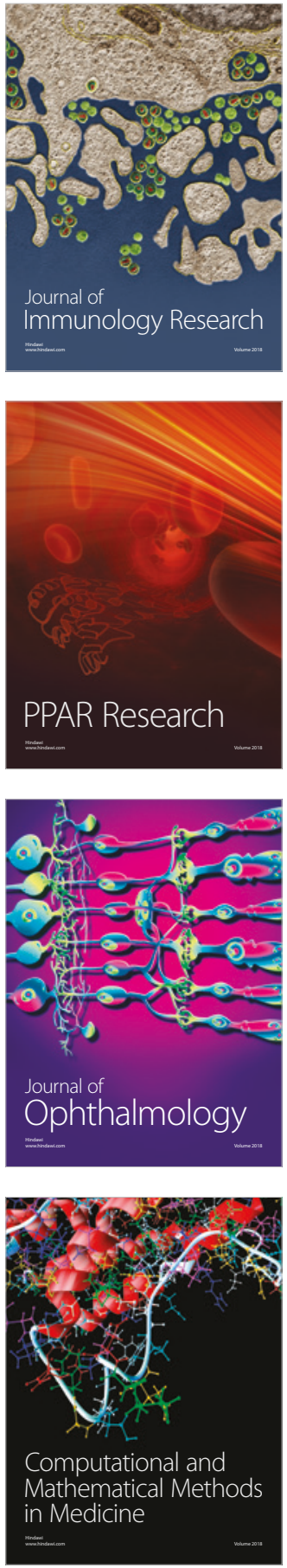

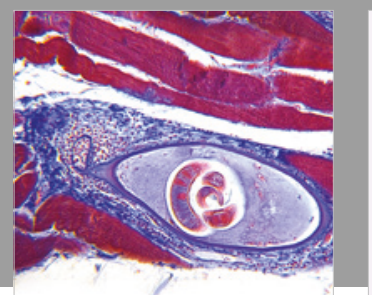

Gastroenterology Research and Practice

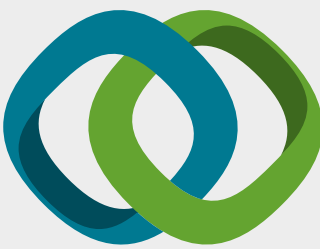

\section{Hindawi}

Submit your manuscripts at

www.hindawi.com
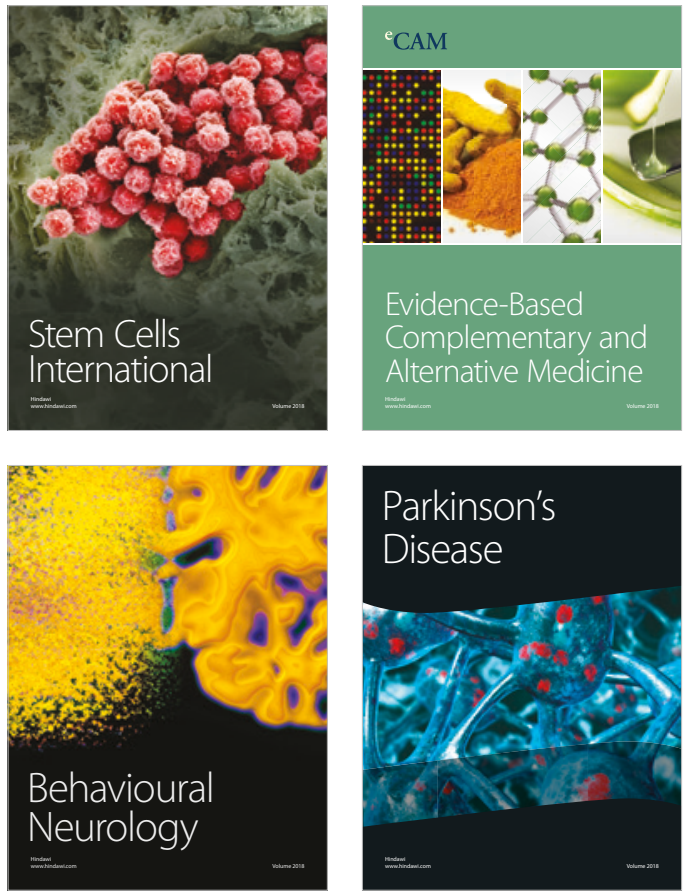

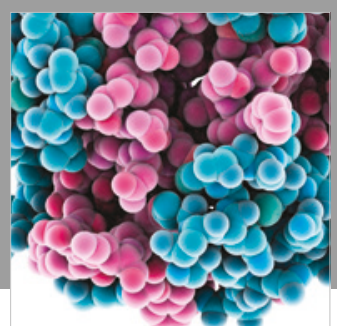

ournal of

Diabetes Research

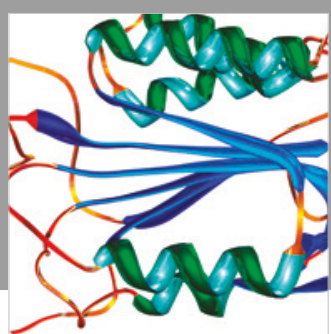

Disease Markers
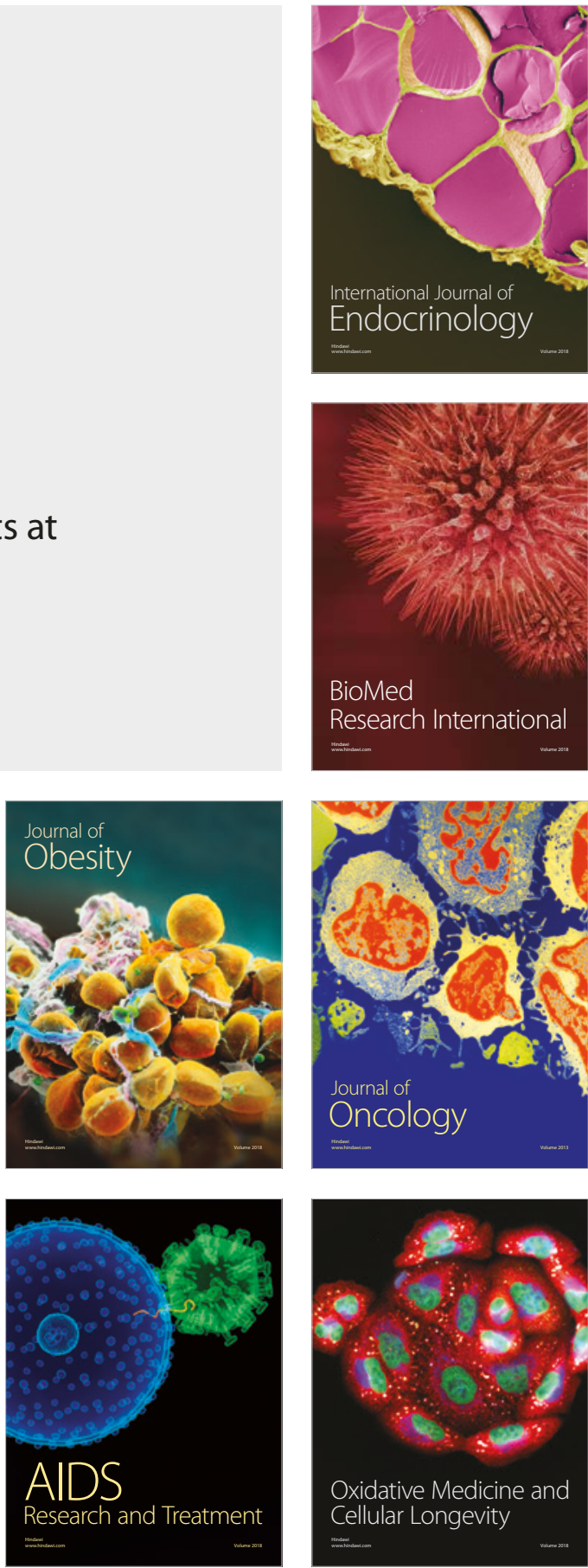